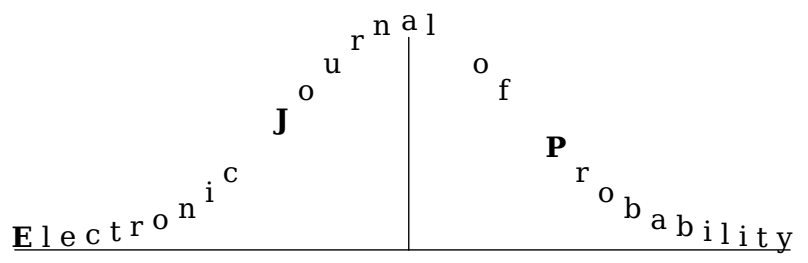

Electron. J. Probab. 26 (2021), article no. 148, 1-25.

ISSN: 1083-6489 https://doi.org/10.1214/21-EJP715

\title{
On the explosion of a class of continuous-state nonlinear branching processes*
}

\author{
Bo $\mathrm{Li}^{\dagger} \quad$ Xiaowen $\mathrm{Zhou}^{\ddagger}$
}

\begin{abstract}
In this paper, we consider a class of generalized continuous-state branching processes obtained by Lamperti type time changes of spectrally positive Lévy processes using different rate functions. When explosion occurs to such a process, we show that the process converges to infinity in finite time asymptotically along a deterministic curve, and identify the speed of explosion for rate function in different regimes. To prove the main theorems, we also establish a new asymptotic result for scale function of the spectrally positive Lévy process.
\end{abstract}

Keywords: continuous-state branching process; spectrally positive Lévy process; Lamperti transform; explosion.

MSC2020 subject classifications: 60J80; 60J50.

Submitted to EJP on December 23, 2020, final version accepted on October 6, 2021.

Supersedes arXiv: 1910.05914v1.

\section{Introduction}

A continuous-state branching process is a nonnegative real-valued Markov process satisfying the additive branching property. It arises as time-space scaling limit of discrete Bienaymé-Galton-Watson processes. On the other hand, it can also be obtained by the Lamperti time change of a spectrally positive Lévy process stopped at hitting 0 for the first time. We refer to [24] and Chapter 12 of [18] for nice introductions on continuous-state branching processes.

The classical Bienaymé-Galton-Watson branching processes had been generalized to those with nonadditive branching mechanism; see for example, [25], [15], [7] and [8]. In the same spirit, continuous-state branching processes with nonadditive branching have been proposed in recent years. In particular, the continuous-state polynomial branching

\footnotetext{
${ }^{*}$ Bo Li and Xiaowen Zhou are supported by NSERC (RGPIN-2016-06704). Xiaowen Zhou is supported by NSFC (\#12171405).

${ }^{\dagger}$ School of Mathematics and LPMC, Nankai University, Tianjin 300071, PR China. E-mail: libo@nankai.edu. $\mathrm{cn}$

${ }^{\ddagger}$ Department of Mathematics and Statistics, Concordia University, Montreal, Canada H3G 1M8. Email: xiaowen.zhou@concordia.ca
} 
process is introduced in [21] as the unique nonnegative solution to a generalized version of the stochastic differential equation in [9], which can be identified as a continuous-state branching process with nonadditive, population dependent branching mechanism. The behaviors of extinction, explosion and coming down from infinity for such a process are discussed in [21]. A more general class of continuous-state branching processes is proposed in [12] via Lamperti type time change of stopped spectrally positive Lévy processes using rate functions $R$ defined on $(0, \infty)$, where the classical continuous-state branching process corresponds to the linear rate function of $R(x)=x$ and the model in [21] corresponds to the rate function of $R(x)=x^{\theta}$. The above continuous-state nonlinear branching processes are further generalized in [22] as solutions to more general versions of the Dawson-Li equation.

For the continuous-state nonlinear branching processes, on one hand, the nonadditive branching mechanism allows richer boundary behaviors such as coming down from infinity; on the other hand, many classical techniques based on the additive branching property fail to work. Criteria for extinction, explosion and coming down from infinity are developed in [21], [22] and [12] for the respective continuous-state nonlinear branching processes via a martingale approach and fluctuation theory for spectrally positive Lévy processes.

The speed of coming down from infinity for such processes is studied in [12] by analyzing the asymptotic behaviors of weighted occupation times for the associated spectrally positive Lévy process. Sufficient conditions are found under which the continuous-state nonlinear branching process comes down from infinity along a deterministic curve.

For the continuous-state nonlinear branching processes introduced in [12], explosion occurs when the process $X$ has a positive drift and the rate function increases fast enough near infinity. In this paper we study the explosion behaviors for such a continuous-state branching process $X$. In particular, we identify the speed of explosion that is defined as the asymptotic of $X\left(T_{\infty}^{+}-t\right)$ as $t \rightarrow 0+$ for the explosion time $T_{\infty}^{+}$. We are not aware of any previous results on the speed of explosion for general Markov processes or for solutions to general stochastic differential equations with jumps. In addition, when explosion happens, using techniques from [19] we also express the potential measure of the process $X$ using the generalized scale functions for the associated spectrally negative Lévy process.

To find the speed of explosion, we treat separately two classes of rate functions, the so called slow regime of rate functions that are perturbations of power functions and the fast regime of rate functions that are perturbations of exponential functions. Our approach relies on analyzing the weighted occupation time for spectrally positive Lévy process. For the process $X$ with rate function from the slow regime, given the explosion occurs we can show that the normalization of random variable $T_{\infty}^{+}-T_{x}^{+}$converges to 1 in the conditional probability, where $T_{x}^{+}$denotes the first upcrossing time of level $x$. Similarly, if the rate function belongs to the fast regime, under the conditional probability of explosion the random variable $T_{\infty}^{+}-T_{x}^{+}$, after rescaling, converges in distribution to a random variable whose distribution can be specified using functionals of spectrally positive Lévy process. The convergence results in both cases lead to an asymptotic on the running maximum of the process near the explosion time. By comparing values of the associated spectrally positive Lévy process with its running maximum, we can show that for rate functions in both regimes the explosion occurs in an asymptotically deterministic fashion. In particular, in the fast regime the speed of explosion is asymptotically proportional to $-\log t$ as time $t \rightarrow 0+$.

Some parts of our approach resemble those in [12] and in [1] for studying the coming down from infinity behaviors of the respective processes. But an additional difficulty emerges in our work due to the overshoot when the nonlinear branching process first 
upcrosses a level $x$ at time $T_{x}^{+}$. We remark that this difficulty seems to be essential and we do not see schemes such as time reversal can easily get around the problem caused by overshoot.

To overcome this difficulty, for the associated spectrally positive Lévy process we identify the Laplace transform of its stationary overshoot distribution, and we obtain a new asymptotic result on the corresponding scale function. For the case of fast regime, instead of showing the convergence of Laplace transform for the weighted occupation time as in [12], we apply the occupation density theorem to the weighted occupation time and the properties of regularly varying functions to show the almost sure convergence that eventually leads to the desired convergence in law.

We also want to point out that our condition on rate function, $\mathrm{H}_{2}$ in Section 3 , for the convergences of the rescaled explosion time can be more general than those in [12].

The rest of the paper is arranged as follows. In Section 2 we first introduce some preliminary results on spectrally positive Lévy processes and the associated scale functions together with the exit problems and the weighted occupation times. The continuous-state nonlinear branching processes are also defined via the Lamperti type transforms in this section. The main results are presented in Section 3. All the proofs are deferred to Section 4. Several intermediate results are also posed and proved in Section 4.

\section{Spectrally positive Lévy processes and continuous-state nonlin- ear branching processes}

Let $\xi$ be a spectrally positive Lévy process (SPLP), that is, a real-valued stochastic process with stationary independent increments and with no negative jumps, defined on a filtered probability space $\left(\Omega, \mathscr{F},\left(\mathscr{F}_{t}\right)_{t \geq 0}, \mathbb{P}\right)$. Its Laplace exponent is well-defined and of the Lévy-Khintchine form, i.e. for $s \geq 0$,

$$
\begin{aligned}
\psi(s) & :=t^{-1} \log \mathbb{E}\left[\exp \left(-s \xi_{t}\right)\right] \\
& =\frac{\sigma^{2}}{2} s^{2}-\mu s+\int_{0}^{\infty}\left(e^{-s x}-1+s x \mathbf{1}(x<1)\right) \Pi(d x),
\end{aligned}
$$

where $\mu \in \mathbb{R}, \sigma \geq 0$ and the Lévy measure $\Pi$ is a $\sigma$-finite measure on $(0, \infty)$ such that $\int_{0}^{\infty}\left(1 \wedge x^{2}\right) \Pi(d x)<\infty$. It is well-known that $\psi(\cdot)$ is continuous and strictly convex on $[0, \infty)$, its right inverse is defined by $\Phi(t):=\sup \{s \geq 0, \psi(s)=t\}$.

Denote by $\mathbb{P}_{x}$ the probability law of $\xi$ for $\xi_{0}=x$, and write $\mathbb{P}$ when $\xi_{0}=0$. We denote throughout this paper

$$
p:=\Phi(0) \quad \text { and } \quad \gamma:=\mathbb{E}\left[\xi_{1}\right]=-\psi^{\prime}(0) .
$$

Notice that $\gamma<\infty$ if and only if $\int_{0}^{\infty}\left(x \wedge x^{2}\right) \Pi(d x)<\infty$, and in this case $\gamma=\mu+\int_{1}^{\infty} x \Pi(d x)$. If $p>0$, then $\psi^{\prime}(0) \in[-\infty, 0), \xi$ is transient and goes to $\infty$ as $t \rightarrow \infty$, and the following result holds.

Lemma 2.1. Write $\bar{\xi}_{t}:=\sup _{0 \leq s \leq t} \xi_{s}$ for the running maximum process of $\xi$. For $p>0$ we have

$$
\xi_{t} / \bar{\xi}_{t} \underset{t \rightarrow \infty}{\longrightarrow} 1 \text { and } \inf _{s>t} \xi_{s} / \xi_{t} \underset{t \rightarrow \infty}{\longrightarrow} 1 \quad \mathbb{P} \text {-a.s. }
$$

Remark 2.2. If $\psi^{\prime}(0)=0$, then $\xi$ oscillates and $\lim \sup _{t \rightarrow \infty} \xi_{t} / \bar{\xi}_{t}=1$ P-a.s.

For $q \geq 0$, the $q$-scale function $W^{(q)}$ is a continuous and increasing function on $[0, \infty)$ with $W^{(q)}(x)=0$ for $x<0$, which satisfies

$$
\int_{0}^{\infty} e^{-s y} W^{(q)}(y) d y=\frac{1}{\psi(s)-q} \text { for } s>\Phi(q) .
$$


We write $W(x) \equiv W^{(0)}(x)$ when $q=0$. Define the first passage times of $\xi$ as

$$
\tau_{x}^{-}:=\inf \left\{t>0, \xi_{t}<x\right\} \text { and } \tau_{x}^{+}:=\inf \left\{t>0, \xi_{t}>x\right\},
$$

with the convention $\inf \emptyset=\infty$. Given the scale function, the following first passage results can be found in Sections 8.1 and 8.2 of [18], for $q \geq 0$ and $c<x<b$

$$
\mathbb{E}_{x}\left[e^{-q \tau_{c}^{-}} ; \tau_{c}^{-}<\tau_{b}^{-}\right]=\frac{W^{(q)}(b-x)}{W^{(q)}(b-c)} \text { and } \quad \mathbb{E}_{x}\left[e^{-q \tau_{c}^{-}}\right]=e^{-\Phi(q)(x-c)},
$$

with the convention $e^{-\infty}=0$. The potential measure of $\xi$ killed upon leaving the interval $[0, \infty)$ is given by

$$
\begin{aligned}
U(x, d y) & :=\int_{0}^{\infty} \mathbb{P}_{x}\left(\xi_{t} \in d y ; t<\tau_{0}^{-}\right) d t \\
& =\left(e^{-p x} W(y)-W(y-x)\right) d y=: u(x, y) d y \quad \text { for } x, y>0 .
\end{aligned}
$$

Change of measure is another useful tool for the fluctuation theory of Lévy processes. For $\alpha \in \mathbb{R}$ with $\psi(\alpha)<\infty$, the process $\left(e^{-\alpha \xi_{t}-\psi(\alpha) t}\right)_{t \geq 0}$ is a martingale under $\mathbb{P}$. Define the probability measure $\mathbb{P}^{(\alpha)}$ by

$$
\left.\frac{d \mathbb{P}^{(\alpha)}}{d \mathbb{P}}\right|_{\mathscr{F}_{t}}=e^{-\alpha \xi_{t}-\psi(\alpha) t} \text { for } t>0
$$

It is well-known that $\xi$ is still a SPLP under $\mathbb{P}^{(\alpha)}$. The associated Laplace exponent and scale functions under $\mathbb{P}^{(\alpha)}$ are denoted similarly with subscripts $\alpha$. A direct calculation shows that

$$
\psi_{\alpha}(s)=\psi(\alpha+s)-\psi(\alpha) \text { and } \Phi_{\alpha}(s)=\Phi(\psi(\alpha)+s)-\alpha \text { for } s \geq 0,
$$

and $W_{\alpha}^{(q)}(x)=e^{-\alpha x} W^{(q+\psi(\alpha))}(x)$. In particular, for the 0-scale function under $\mathbb{P}^{(p)}$

$$
W_{p}(y)=e^{-p y} W(y) \uparrow W_{p}(\infty)=\Phi^{\prime}(0) .
$$

We refer to [17] and [13] for a more detailed discussions and examples of scale functions.

The following limiting result on the resolvent density in (2.3) is useful in this paper, and we refer to Theorem I.21 of [2] for a similar result called "renewal theorem".

Lemma 2.3. If $p, \gamma \in(0, \infty)$, we have

$$
\lim _{y \rightarrow \infty}\left(e^{p y} \Phi^{\prime}(0)-W(y)\right)=\gamma^{-1} .
$$

A direct consequence of the limit above is that for any $k \in \mathbb{R}$,

$$
\begin{aligned}
& \lim _{y \rightarrow \infty} \sup _{x \geq k}\left|u(x, x+y)-\frac{1-e^{-p x}}{\gamma}\right| \\
& =\lim _{y \rightarrow \infty} \sup _{x \geq k}\left|\left(e^{-p x} W(x+y)-W(y)\right)-\frac{1-e^{-p x}}{\gamma}\right|=0 .
\end{aligned}
$$

Remark 2.4. By change of measure, we obtain the following general result where a light-tailed condition on $\Pi$ is required. For $q \geq 0$, let $\phi(q)$ be the left-root of $t \rightarrow \psi(t)-q$ and assume $\psi^{\prime}(\phi(q)) \in(-\infty, 0)$, i.e. $\phi(q)<\Phi(q)$ with $\psi(\phi(q))=q$, then $\phi(q) \leq 0$ and

$$
e^{-\phi(q) y}\left(e^{-\Phi(q) x} W^{(q)}(x+y)-W^{(q)}(y)\right) \underset{y \rightarrow \infty}{\longrightarrow} \frac{e^{(\phi(q)-\Phi(q)) x}-1}{\psi^{\prime}(\phi(q))},
$$

where $-\phi(q)$ is also known as the unique nonnegative root of the Cramér-Lundberg equation $\psi(-t)=q$ in risk theory. 
Lemma 2.3 is proved based on the following result used in [10]; see also Theorem 5.7 of [18] and [3]. If $\gamma \in(0, \infty)$, then

$$
\mathbb{P}\left(\xi\left(\tau_{y}^{+}\right)-y \in d z\right) \underset{y \rightarrow \infty}{\Longrightarrow} \rho(d z),
$$

for some non-degenerate weak limit $\rho$ on $[0, \infty)$, called the stationary overshoot distribution in [10], which is characterized in the following lemma, see also Lemma 3 of [4] for Lévy process in a half-line.

Lemma 2.5. If $p, \gamma \in(0, \infty)$, we have for $s \geq 0$

$$
\widehat{\rho}(s):=\int_{0-}^{\infty} e^{-s z} \rho(d z)=\frac{p \psi(s)}{\gamma s(s-p)} .
$$

In particular, $\widehat{\rho}(p)=\left(\gamma \Phi^{\prime}(0)\right)^{-1}$.

The continuous-state nonlinear branching process $X$ considered in this paper is defined in [21] by time changing a spectrally positive Lévy process. More precisely, for a Borel function $R(\cdot)$ on $(0, \infty)$, which is positive and locally bounded away from 0 , define an additive functional

$$
\eta(t):=\int_{0}^{t} \frac{1}{R\left(\xi_{s}\right)} d s \text { for } t<\tau_{0}^{-},
$$

and $\eta(\infty):=\lim _{t \rightarrow \infty} \eta(t)$ on the event $\left\{\tau_{0}^{-}=\infty\right\}$. Its right inverse function is defined as $\eta^{-1}(t):=\inf \{s>0, \eta(s)>t\}$ for $t<\eta\left(\tau_{0}^{-}\right)$. Then the process $X$ is defined, stopped at time $\eta\left(\tau_{0}^{-}\right) \leq \infty$, by letting

$$
X_{t}:=\xi\left(\eta^{-1}(t)\right) \text { for } t \in\left[0, \eta\left(\tau_{0}^{-}\right)\right) .
$$

It is true that $X$ is a well-defined positive-valued Markov process with absorbing states $\{0, \infty\}$. Define the first passage times of $X$ by

$$
T_{x}^{-}:=\inf \left\{t>0, X_{t}<x\right\}, \quad T_{x}^{+}:=\inf \left\{t>0, X_{t}>x\right\},
$$

for $x \in(0, \infty)$ and

$$
T_{0}^{-}:=\lim _{x \rightarrow 0+} T_{x}^{-} \quad \text { and } \quad T_{\infty}^{+}:=\lim _{x \rightarrow \infty} T_{x}^{+},
$$

with the convention $\inf \emptyset=\infty$. The following identities on the first passage times follow immediately from the Lamperti type transform. For any $x>0$ we have

$$
T_{x}^{+}=\eta\left(\tau_{x}^{+}\right) \text {on the event }\left\{\tau_{x}^{+}<\tau_{0}^{-}\right\} \text {and } T_{x}^{-}=\eta\left(\tau_{x}^{-}\right) \text {on the event }\left\{\tau_{x}^{-}<\infty\right\} .
$$

In addition, for the absorbing time $\eta\left(\tau_{0}^{-}\right)$, we have

$$
\eta\left(\tau_{0}^{-}\right)=\left\{\begin{array}{lll}
T_{0}^{-}=\eta\left(\tau_{0}^{-}\right) & \text {on the event }\left\{\tau_{0}^{-}<\infty\right\} \\
T_{\infty}^{+}=\eta(\infty) & \text { on the event }\left\{\tau_{0}^{-}=\infty\right\}
\end{array}\right.
$$

More precisely, at $\eta\left(\tau_{0}^{-}\right)$, the process $X$ becomes extinct at the finite time $T_{0}^{-}=\eta\left(\tau_{0}^{-}\right)$ with $X\left(T_{0}^{-}\right)=0$ on the event $\left\{\eta\left(\tau_{0}^{-}\right)<\infty, \tau_{0}^{-}<\infty\right\}$; it becomes extinguishing when $\lim _{t \rightarrow \infty} X(t)=0$ on the event $\left\{\eta\left(\tau_{0}^{-}\right)=\infty, \tau_{0}^{-}<\infty\right\}$; it explodes at the finite time $T_{\infty}^{+}=\eta(\infty)$ with $X\left(T_{\infty}^{+}\right)=\infty$ on the event $\left\{\eta(\infty)<\infty, \tau_{0}^{-}=\infty\right\}$; and it drifts to infinity when $\lim _{t \rightarrow \infty} X(t)=\infty$ on the event $\left\{\eta(\infty)=\infty, \tau_{0}^{-}=\infty\right\}$. $T_{0}^{-}$is called the extinction time of $X$ if $T_{0}^{-}<\infty$, and $T_{\infty}^{+}$is called the explosion time of $X$ if $T_{\infty}^{+}<\infty$.

We first characterize the extinction and explosion conditions for the process $X$ using integral tests. Note that similar results are obtained in [21] for power function $R$. 
Proposition 2.6. Extinction occurs for the process $X$ with a positive probability, i.e.

$$
\mathbb{P}_{x}\left(\eta\left(\tau_{0}^{-}\right)<\infty, \tau_{0}^{-}<\infty\right)>0 \text { if and only if } \int_{0+} \frac{W(z)}{R(z)} d z<\infty .
$$

Moreover, in this case, $\mathbb{P}_{x}\left(\eta\left(\tau_{0}^{-}\right)<\infty \mid \tau_{0}^{-}<\infty\right)=1$ and the extinction probability is $\mathbb{P}_{x}\left(T_{0}^{-}<\infty\right)=e^{-p x}$ for all $x>0$.

If $p, \gamma \in(0, \infty)$, the process $X$ explodes with a positive probability, i.e.

$$
\mathbb{P}_{x}\left(\eta(\infty)<\infty, \tau_{0}^{-}=\infty\right)>0 \quad \text { if and only if } \int^{\infty} \frac{1}{R(z)} d z<\infty .
$$

Moreover, in this case, $\mathbb{P}_{x}\left(\eta(\infty)<\infty \mid \tau_{0}^{-}=\infty\right)=1$ and the explosion probability is $\mathbb{P}_{x}\left(T_{\infty}^{+}<\infty\right)=1-e^{-p x}$ for all $x>0$.

We remark here that by definition of explosion time in (2.10), the first passage identities (2.11), (2.12) and the boundary behaviors for process $X$ specified thereafter, the event of explosion is equivalent to the finiteness of the so-called perpetual integrals of spectrally negative Lévy processes on the set $\left\{\tau_{0}^{-}=\infty\right\}$, which has been studied under different conditions; c.f. $[10,23,16]$ and the references therein.

In the paper, we first introduce the following assumptions on the rate function $R$.

$$
\mathbb{H}_{0}: \int^{\infty} \frac{d y}{R(y)}<\infty \quad \text { and } \mathbb{H}_{1}: \int_{0+}^{\infty} \frac{W_{p}(y)}{R(y)} d y<\infty \text { for } p \geq 0 .
$$

By Proposition 2.6, condition $\mathrm{H}_{0}$ can be referred to as the explosion condition, under which we denote by

$$
\varphi(x):=\frac{1}{\gamma} \int_{x}^{\infty} \frac{d y}{R(y)} \text { for } x>0 .
$$

Remark 2.7. Observe that condition $\mathbb{H}_{1}$ implies condition $\mathbb{H}_{0}$. Further, if $p>0$, then $W_{p}(\infty)<\infty$ and the condition $\mathbb{H}_{1}$ is equivalent to

$$
\int_{0+}^{1} \frac{W(y)}{R(y)} d y+\int_{1}^{\infty} \frac{1}{R(y)} d y<\infty
$$

which, although stronger than the explosion condition $\mathbb{H}_{0}$, allows to find explicit expressions for general $R$ for further analysis; c.f. Corollary 4.5, Remarks 4.6 and 4.8.

Remark 2.8. For a general rate function $R,\left\{T_{\infty}^{+}<\infty\right\}=\left\{T_{\infty}^{+}<T_{0}^{-}\right\}$,

$$
\left\{T_{0}^{-}=\infty\right\}=\left\{\tau_{0}^{-}=\infty\right\} \cup\left(\left\{\tau_{0}^{-}<\infty\right\} \cap\left\{\eta\left(\tau_{0}^{-}\right)=\infty\right\}\right),
$$

and

$$
\left\{T_{\infty}^{+}=\infty\right\}=\left\{\tau_{0}^{-}<\infty\right\} \cup\left(\left\{\tau_{0}^{-}=\infty\right\} \cap\{\eta(\infty)=\infty\}\right) .
$$

We may have $\mathbb{P}\left(\left\{T_{\infty}^{+}=\infty\right\} \cap\left\{T_{0}^{-}=\infty\right\}\right)>0$ for some $R$. In such a case, there is a positive probability that the process $\xi$ either reaches 0 or goes to $\infty$ before reaching 0 , where the associated $X$ either extinguishes or drifts to $\infty$ respectively. Proposition 2.6 shows that, under condition $\mathbb{H}_{0}$ we have $\left\{T_{\infty}^{+}<\infty\right\}=\left\{\tau_{0}^{-}=\infty\right\}$, and under stronger condition $\mathbb{H}_{1}$, we have $\left\{T_{0}^{-}<\infty\right\}=\left\{\tau_{0}^{-}<\infty\right\}$, which gives $\mathbb{P}\left(\left\{T_{\infty}^{+}<\infty\right\} \cup\left\{T_{0}^{-}<\infty\right\}\right)=1$.

Remark 2.9. By Proposition VII.10 in [2], $W_{p}(x) \asymp \frac{1}{x \psi_{p}(1 / x)}$, we have

$$
\int_{0+} \frac{W(z)}{R(z)} d z<\infty \text { if and only if } \int^{\infty} \frac{1}{z \psi_{p}(z) R(1 / z)} d z<\infty
$$

and

$$
\int^{\infty} \frac{W_{p}(z)}{R(z)} d z<\infty \text { if and only if } \int_{0+} \frac{1}{z \psi_{p}(z) R(1 / z)} d z<\infty
$$


In particular, for $R(z)=z^{\theta}$, by change of variable, we have from Proposition 2.6 that

$$
\mathbb{P}_{x}\left(T_{0}^{-}<\infty\right)>0 \text { if and only if } \int^{\infty} \frac{z^{\theta-1}}{\psi_{p}(z)} d z<\infty,
$$

which coincides with Theorem 1.8 of [21]. If $p, \gamma \in(0, \infty)$, then

$$
\mathbb{P}_{x}\left(T_{\infty}^{+}<\infty\right)>0 \text { if and only if } \theta>1,
$$

which coincides with Theorem 1.10(1) of [21].

\section{Main results}

Under the condition $\mathbb{H}_{1}$, the explosion time $T_{\infty}^{+}$has finite exponential moment.

Theorem 3.1. Assume $p>0$ and the condition $\mathbb{H}_{1}$ holds for function $R$. Then $m_{n}(x):=$ $\mathbb{E}_{x}\left[\left(T_{\infty}^{+}\right)^{n} ; T_{\infty}^{+}<T_{0}^{-}\right]$is finite and can be obtained recursively by

$$
m_{n}(x)=n \int_{0}^{\infty} u(x, y) \frac{m_{n-1}(y)}{R(y)} d y \quad \text { with } \quad m_{0}(x)=1-e^{-p x}
$$

where $u$ is the potential density in (2.3). For $|q|<\left(\int_{0+}^{\infty} \frac{W_{p}(z)}{R(z)} d z\right)^{-1}$, we have for $x>0$

$$
\mathbb{E}_{x}\left[e^{q \cdot T_{\infty}^{+}} ; T_{\infty}^{+}<T_{0}^{-}\right]=\sum_{n=0}^{\infty} \frac{q^{n}}{n !} m_{n}(x)<\infty .
$$

Recall $p, \gamma$ defined in (2.1) and $\varphi$ defined in (2.13). To study the asymptotic behavior of the process $X$ near time $T_{\infty}^{+}$on $\left\{T_{\infty}^{+}<\infty\right\}$, we always assume $p, \gamma \in(0, \infty)$, the explosion condition $\mathbb{H}_{0}$ and the following condition hold, for some $\lambda \in[0, \infty)$ and every $x>0$

$$
\mathbb{H}_{2}: \frac{\varphi(x+y)}{\varphi(y)} \underset{y \rightarrow \infty}{\longrightarrow} e^{-\lambda x}
$$

Denote by

$$
\mathbb{Q}_{t}(x, A):=\mathbb{P}_{x}\left(X_{t} \in A\right)=\mathbb{P}_{x}\left(X_{t} \in A, t<T_{0}^{-} \wedge T_{\infty}^{+}\right),
$$

and under condition $\mathbb{H}_{0}$,

$$
\mathbb{Q}_{x}^{\uparrow}(B):=\mathbb{Q}_{x}\left(B \mid T_{\infty}^{+}<\infty\right)=\mathbb{P}_{x}\left(B \mid \tau_{0}^{-}=\infty\right)=: \mathbb{P}_{x}^{\uparrow}(B),
$$

for $x>0, t \geq 0, A \in \mathscr{B}(0, \infty)$ and $B \in \sigma\left\{X_{t}, t \geq 0\right\} \subset \sigma\left\{\xi_{s}, s \geq 0\right\}$. Then $\mathbb{Q}_{t}$ defines the semigroup of $X$ before absorption, $\mathbb{Q}_{x}^{\uparrow}$ defines the probability law of $X$ conditioned on explosion, and $\mathbb{P}_{x}^{\uparrow}$ denotes the probability law of $\xi$ conditioned to stay positive, where under condition $\mathbb{H}_{0}$ and $p>0, \mathbb{Q}_{x}^{\uparrow}$ is meaningful with $\mathbb{Q}_{x}^{\uparrow}=\mathbb{P}_{x}^{\uparrow}$ by Remark 2.8.

Remark 3.2. Recall that, a positive function $f$ on $(0, \infty)$ is regularly varying with index $\alpha \in \mathbb{R}$ at $\infty$ if for any $s>0$,

$$
f(s x) / f(x) \rightarrow s^{\alpha} \quad \text { as } x \rightarrow \infty,
$$

and is slowly varying at $\infty$ if $\alpha=0$.

The condition $\mathbb{H}_{2}$ is equivalent to function $x \rightarrow \varphi(\log x)$ being regularly varying with index $-\lambda \in(-\infty, 0]$. If there exists a positive function $f$ such that

$$
\frac{\varphi(x+y)}{\varphi(y)} \underset{y \rightarrow \infty}{\longrightarrow} f(x) \text { for all } x>0,
$$


then condition $\mathbb{H}_{2}$ necessarily holds; see Theorem 1.4.1 of [5]. Moreover, under condition $\mathrm{H}_{2}$ we have

$$
\log \varphi(x+y)-\log \varphi(y) \underset{y \rightarrow \infty}{\longrightarrow}-\lambda x .
$$

It follows from Lemma 1.4.5 of [5] that

$$
\varphi(x)=e^{-(\lambda+\epsilon(x)) x} \quad \text { for some function } \epsilon \text { satisfying } \epsilon(x) \rightarrow 0 \text { as } x \rightarrow \infty,
$$

which can also be obtained from a representation of regularly varying function.

A sufficient condition for the condition $\mathbb{H}_{2}$ is that function $x \rightarrow R(\log x)$ varies regularly with index $\lambda \geq 0$, which can be proved by applying Karamata's theorem, c.f. Theorem 1.5.11 and Proposition 1.5.9.b of [5]. An interesting example for $R$ is a power-like function satisfying the condition $\mathbb{H}_{0}$ with $\lambda=0$. If

$$
\liminf _{x \rightarrow \infty} x^{\alpha} R(x)>0 \text { and } \limsup _{x \rightarrow \infty} x^{\beta} R(x)<\infty,
$$

for some constants $\alpha \geq \beta$ with $\alpha-\beta<1$, then $\mathbb{H}_{2}$ holds with $\lambda=0$. Actually, under condition $\mathbb{H}_{0}$, we must have $\beta<-1$, thus for some constant $c>0$ and $x$ large enough,

$$
\frac{\varphi(x)-\varphi(x+a)}{\varphi(x)}=\frac{\int_{x}^{x+a} \frac{1}{R(y)} d y}{\int_{x}^{\infty} \frac{1}{R(y)} d y} \leq c \frac{\int_{x}^{x+a} y^{\alpha} d y}{\int_{x}^{\infty} y^{\beta} d y} \leq \frac{-c a}{1+\beta} x^{\alpha-\beta-1} \rightarrow 0 .
$$

We are ready to present our results on explosion whose proofs are deferred to Section 4. Recall from Proposition 2.6 that, if $p, \gamma \in(0, \infty)$ and $\mathbb{H}_{0}$ holds, $\mathbb{Q}_{x}^{\uparrow}\left(T_{\infty}^{+}<\infty\right)=$ 1.

We first present the asymptotic of the residual explosion time after first uncrossing a level.

Theorem 3.3. Suppose that $p, \gamma \in(0, \infty)$ and $R$ satisfies the conditions $\mathbb{H}_{0}$ and $\mathbb{H}_{2}$, and let $\lambda \geq 0$ be the constant in $\mathrm{H}_{2}$.

(A) If $\lambda=0$, then for every $x>0$

$$
\frac{T_{\infty}^{+}-T_{z}^{+}}{\varphi(z)} \rightarrow 1 \text { in } \mathbb{Q}_{x}^{\uparrow} \text { as } z \rightarrow \infty
$$

(B) If $\lambda \in(0, \infty)$ and $\lim \sup _{x \rightarrow \infty} \frac{1}{\varphi^{2}(x)} \int_{x}^{\infty} \frac{1}{R^{2}(y)} d y<\infty$, then as $z \rightarrow \infty$

$$
\begin{gathered}
\left.\frac{\varphi\left(X\left(T_{z}^{+}\right)\right)}{\varphi(z)}\right|_{\mathbb{Q}_{x}^{\uparrow}} \stackrel{D}{\Rightarrow} e^{-\lambda \varrho},\left.\quad \frac{T_{\infty}^{+}-T_{z}^{+}}{\varphi\left(X\left(T_{z}^{+}\right)\right)}\right|_{\mathbb{Q}_{x}^{\uparrow}} \stackrel{D}{\Rightarrow} \lambda \gamma \int_{0}^{\infty} e^{-\lambda \xi_{t}} d t \\
\text { and }\left.\quad \frac{T_{\infty}^{+}-T_{z}^{+}}{\varphi(z)}\right|_{\mathbb{Q}_{x}^{\uparrow}} \stackrel{D}{\Rightarrow} \lambda \gamma e^{-\lambda \varrho} \int_{0}^{\infty} e^{-\lambda \xi_{t}} d t,
\end{gathered}
$$

where $\left.Z\right|_{\mathbb{Q}_{x}^{\uparrow}}$ denotes the law of $Z$ under $\mathbb{Q}_{x}^{\uparrow}$, and where $\varrho$ is a random variable independent of $\xi$ with probability law $\rho$ specified in (2.7).

Remark 3.4. By (4.14) in the proof of Theorem 3.3 we see that, under conditions $\mathbb{H}_{1}$ and $\mathbb{H}_{2}$, the stronger $L^{2}\left(\mathbb{Q}_{x}^{\uparrow}\right)$ convergence holds for assertion (A) of Theorem 3.3, that is,

$$
\lim _{z \rightarrow \infty} \mathbb{Q}_{x}^{\uparrow}\left[\left(\frac{T_{\infty}^{+}-T_{z}^{+}}{\varphi(z)}-1\right)^{2}\right]=0 .
$$

Remark 3.5. If $x \rightarrow R(\log x)$ is a regularly varying function with index $\lambda>0$ as $x \rightarrow \infty$, it follows from Karamata's theorem that

$$
\int_{x}^{\infty} \frac{R^{2}(x)}{R^{2}(y)} d y \rightarrow(2 \lambda)^{-1} \text { and } \quad \int_{x}^{\infty} \frac{R(x)}{R(y)} d y \rightarrow \lambda^{-1} \quad \text { as } \quad x \rightarrow \infty
$$


thus,

$$
\frac{1}{\varphi^{2}(x)} \int_{x}^{\infty} \frac{1}{R^{2}(y)} d y \rightarrow \frac{\gamma^{2} \lambda}{2} .
$$

In particular, if $R(x) e^{-\lambda x}$ is a regularly varying function, then $x \rightarrow R(\log x)$ is regularly varying with index $\lambda$.

We now present the following main result concerning the speed of explosion.

Theorem 3.6. Suppose that $p, \gamma \in(0, \infty)$ and $R$ satisfies the conditions $\mathbb{H}_{0}$ and $\mathbb{H}_{2}$.

(a) If $\lambda=0$ and $\liminf _{y \rightarrow \infty} \frac{\varphi(y)}{\varphi(h y)} \in(1, \infty]$ for every $h>1$, then we have for every $x>0$

$$
\left.\frac{X\left(T_{\infty}^{+}-t\right)}{\varphi^{-1}(t)}\right|_{\mathbb{Q}_{x}^{\uparrow}} \rightarrow 1 \text { and }\left.\quad \frac{\inf _{0<s<t} X\left(T_{\infty}^{+}-s\right)}{\varphi^{-1}(t)}\right|_{\mathbb{Q}_{x}^{\uparrow}} \rightarrow 1 \text { as } t \rightarrow 0+,
$$

where $\varphi^{-1}(t):=\sup \{s>0, \varphi(s)>t\}$ is the right inverse of $\varphi$.

(b) If $\lambda>0$ and $\lim _{x \rightarrow \infty} \frac{1}{\varphi(x)^{2}} \int_{x}^{\infty} \frac{1}{R^{2}(y)} d y<\infty$, then we have

$$
\left.\frac{X\left(T_{\infty}^{+}-t\right)}{-\log t}\right|_{\mathbf{Q}_{x}^{\uparrow}} \rightarrow \lambda^{-1} \text { and }\left.\frac{\inf _{0<s<t} X\left(T_{\infty}^{+}-s\right)}{-\log t}\right|_{\mathbf{Q}_{x}^{\uparrow}} \rightarrow \lambda^{-1} \quad \text { as } t \rightarrow 0+.
$$

Remark 3.7. If for some $M, m>0$ and $\alpha>1, m x^{\alpha}<R(x)<M x^{\alpha}$ for all $x$ large enough, then for every $h>1$ we have

$$
\frac{\varphi(x)-\varphi(x h)}{\varphi(x)}=\frac{\int_{x}^{x h} \frac{1}{R(y)} d y}{\int_{x}^{\infty} \frac{1}{R(y)} d y} \geq \frac{m \int_{x}^{x h} y^{-\alpha} d y}{M \int_{x}^{\infty} y^{-\alpha} d y}=\frac{m}{M}\left(1-h^{1-\alpha}\right)>0,
$$

and the corresponding function $R$ satisfies the condition (a) in Theorem 3.6.

Remark 3.8. For the asymptotic functions in Theorems 3.3 and 3.6,

- if $R(x)=(c+x)^{\theta}$ for $\theta>1$ and any constant $c>0$, then

$$
\varphi(x)=\frac{(x+c)^{1-\theta}}{\gamma(\theta-1)} \quad \text { and } \quad \varphi^{-1}(t) \sim(\gamma(\theta-1) t)^{\frac{1}{1-\theta}} \quad \text { as } \quad t \rightarrow 0+
$$

- if $R(x)=e^{\lambda x}$ for $\lambda>0$, then $\varphi(x)=(\lambda \gamma)^{-1} e^{-\lambda x}$ and $\varphi^{-1}(t) \sim-\lambda^{-1} \log t$ as $t \rightarrow 0+$.

Remark 3.9. Studying the explosion behaviors of $X$ for rate function $R$ with arbitrary behavior near $\infty$ seems to be rather challenging since the explosion may allow different speeds when the explosion time is approached in different ways. To this end, we assume $\mathrm{H}_{2}$ on the asymptotic behavior of the rate function, which is similar to those assumptions in [1] and [12].

Remark 3.10. We assume that $\gamma \in(0, \infty)$ in both Theorem 3.3 and Theorem 3.6. It remains open to identify the speed of explosion for continuous-state nonlinear processes with big jumps in the sense that $\int_{1}^{\infty} x \Pi(d x)=\infty$.

\section{Proofs}

This Section is dedicated to the proofs of the main results. Lemmas 2.1, 2.3 and 2.5 for SPLP are of independent interest and are proved first. They will be applied in the proofs of main results thereafter. Recall $p$ and $\gamma$ defined in (2.1). 


\subsection{Proofs of Lemmas 2.1, 2.3 and 2.5}

Our proof of Lemma 2.1 is based on the Itô excursion theory, where the compensation formula and the exponential formula for Poisson point process are applied; c.f. Chapter $\mathrm{O}$ of [2]. Here we use the standard notions in the fluctuation theory of Lévy process from [2]. Let $\chi:=\bar{\xi}-\xi$ be the Lévy process reflected at its running maximum, where $\bar{\xi}_{t}=\sup _{s<t} \xi_{s}$ is the running maximum process of $\xi$. Let $l$ be a local time process of $\chi$ at 0 and $l^{-1}$ be its right inverse. Since $\lim _{t \rightarrow \infty} \xi(t)=\infty, \chi$ is a recurrent Markov process. In addition, $\left(l_{s}^{-1}, \xi\left(l_{s}^{-1}\right)\right)_{s>0}$ defines a proper bivariate subordinator on $(0, \infty)$, called the ladder process in Chapter VI of [2], with a version of its Laplace exponent given by $\widehat{\kappa}(\alpha, \beta)=\frac{\alpha-\psi(\beta)}{\Phi(\alpha)-\beta}$. The excursion process $\left(\epsilon_{s}\right)_{s \geq 0}$ of $\chi$ away from 0 , defined by

$$
\epsilon_{s}:=\left\{\begin{array}{cc}
\left\{\chi\left(t+l_{s-}^{-1}\right), 0 \leq t<l_{s}^{-1}-l_{s-}^{-1}\right\} & \text { if } l_{s-}^{-1}<l_{s}^{-1} \\
\Delta & \text { otherwise }
\end{array}\right.
$$

for some isolated point $\Delta$, is a Poisson point process with characteristic measure $n$. The associated excursion height process is denoted by $\left(\bar{\epsilon}_{s}\right)_{s \geq 0}$, that is,

$$
\bar{\epsilon}_{s}:=\left\{\begin{array}{cc}
\sup _{l_{s-}^{-1}<t<l_{s}^{-1}} \chi(t) & \text { if } l_{s-}^{-1}<l_{s}^{-1}, \\
\Delta & \text { otherwise. }
\end{array}\right.
$$

Then $\left(\bar{\epsilon}_{s}\right)_{s \geq 0}$ is a $\mathbb{R}_{+}$-valued Poisson point process and we occasionally drop index $s$.

Proof of Lemma 2.1. Assume $p>0$, then $\mathbb{P}\left(\tau_{1 / \varepsilon}^{+}<\infty\right)=1$ for every $\varepsilon \in(0,1)$. If $t>\tau_{1 / \varepsilon}^{+}$ and $\bar{\xi}(t)-\xi(t) \geq \varepsilon \cdot \bar{\xi}(t)$, we have $t \in\left(l^{-1}(s-), l^{-1}(s)\right)$ with $s=l(t)$. Then we have for this excursion $\epsilon, \bar{\epsilon}_{s}>1$ and $\bar{\epsilon}_{s}>\varepsilon \cdot \bar{\xi}(t)=\varepsilon \cdot \bar{\xi}\left(l_{s-}^{-1}\right)$. Therefore, counting the number of those excursions gives

$$
\left\{\varepsilon<\limsup _{t \rightarrow \infty} \frac{\bar{\xi}(t)-\xi(t)}{\bar{\xi}(t)}\right\}=\left\{\#\left\{s>0 \mid \bar{\epsilon}_{s}>1, \bar{\epsilon}_{s}>\varepsilon \cdot \bar{\xi}\left(l_{s-}^{-1}\right)\right\}:=N_{\varepsilon}=\infty\right\} .
$$

On the other hand, since the process $\chi$ has no positive jumps, the law of $\bar{\epsilon}$ given $\bar{\epsilon}>1$ under $n(\cdot)$ is identical to the law of $\left|\inf _{t<\tau_{0}^{+}} \xi(t)\right|$ under $\mathbb{P}_{-1}$, that is, for $y>1$

$$
n(\bar{\epsilon}>y \mid \bar{\epsilon}>1)=\mathbb{P}_{-1}\left(\inf _{t<\tau_{0}^{+}} \xi(t)<-y\right)=\mathbb{P}_{-1}\left(\tau_{-y}^{-}<\tau_{0}^{+}\right)=\frac{W(1)}{W(y)} .
$$

Notice that similar to Lemma VI.2 of [2], the excursion height process $\left(\bar{\epsilon}_{s}, s>0\right)$ is independent of $\left(\bar{\xi}\left(l_{s-}^{-1}\right), s>0\right)$. Therefore, conditioning on $\left(\bar{\xi}\left(l_{s-}^{-1}\right), s>0\right), N_{\varepsilon}$ is Poisson distributed with parameter

$$
\left(\int_{0}^{\infty} \frac{n(\bar{\epsilon}>1) W(1)}{W\left(1 \vee \varepsilon \cdot \bar{\xi}\left(l_{s-}^{-1}\right)\right)} d s\right)
$$

Since $W(x)=e^{p x} W_{p}(x) \geq W_{p}(1) e^{p x}$ for $x \geq 1$, and P-a.s. $\bar{\xi}\left(l_{s-}^{-1}\right)=\bar{\xi}\left(l_{s}^{-1}\right)=\xi\left(l_{s}^{-1}\right)$ for almost every $s>0$, by the right-continuity of $\xi$ and the definition of $l^{-1}$, we have from Fubini's theorem that

$$
\mathbb{E}\left[\int_{0}^{\infty} \frac{W_{p}(1)}{W\left(1 \vee \varepsilon \cdot \bar{\xi}\left(l_{s-}^{-1}\right)\right)} d s\right] \leq \int_{0}^{\infty} \mathbb{E}\left[e^{-p \varepsilon \cdot \xi\left(l_{s}^{-1}\right)}\right] d s=\frac{p(1-\varepsilon)}{-\psi(\varepsilon p)}<\infty,
$$

where in the equality above we use the fact that $\xi\left(l_{s}^{-1}\right)$ is a subordinator with Laplace exponent $\frac{-\psi(\beta)}{p-\beta}$. Therefore, $\mathbb{P}$ almost surely, we have

$$
\int_{0}^{\infty} \frac{n(\bar{\epsilon}>1) W(1)}{W\left(1 \vee \varepsilon \cdot \bar{\xi}\left(l_{s-}^{-1}\right)\right)} d s<\infty \quad \Leftrightarrow \quad N_{\varepsilon}<\infty \quad \Leftrightarrow \quad \limsup _{t \rightarrow \infty} \frac{\bar{\xi}(t)-\xi(t)}{\bar{\xi}(t)} \leq \varepsilon
$$


and finish the proof of the first assertion.

For the second limit, for every $\varepsilon>0$, suppose $t>0$ such that $\xi_{t} \geq(1+\varepsilon) \inf _{s>t} \xi_{s}>0$. There exists $r>t$ such that $\sqrt{1+\varepsilon} \cdot \inf _{s>t} \xi_{s} \geq \xi_{r}$. Then for this $r$ we have

$$
\sup _{s \leq r} \xi_{s} \geq \xi_{t} \geq(1+\varepsilon) \inf _{s>t} \xi_{s} \geq \sqrt{1+\varepsilon} \cdot \xi_{r} .
$$

The first assertion is then applied to prove the second assertion.

We now prove Lemma 2.5 before Lemma 2.3.

Proof of Lemma 2.5. The proof is based on the observation that $\xi$ and its ladder height process have the same overshoot when first up-crossing a level. Thus, the stationary overshoot is identical in law to the limit of the overshoot of the ladder process, and where we need the assumption of $\gamma=\mathbb{E}\left[\xi_{1}\right] \in(0, \infty)$.

More specifically, consider a ladder height process of $\xi$, which is a subordinator with a version of Laplace exponent $\widehat{\kappa}(\beta)=\frac{\psi(\beta)}{\beta-p}$, c.f. Theorem VII.4 of [2]. Let $\delta$ and $\nu(d z)$ be the associated drift parameter and jump measure, respectively. Then we have from Theorem 5.7 of [18] that

$$
\begin{aligned}
\widehat{\rho}(s) & :=\int_{0-}^{\infty} e^{-s z} \rho(d z)=\int_{0}^{\infty} d y \int_{0}^{\infty} \frac{e^{-s z}}{\mu} \nu(d z+y)+\frac{\delta}{\mu} \\
& =\frac{1}{\mu}\left(\delta+\frac{1}{s} \int_{0}^{\infty}\left(1-e^{-s z}\right) \nu(d z)\right)=\frac{\hat{\kappa}(s)}{\mu s}=\frac{\psi(s)}{\mu s(s-p)},
\end{aligned}
$$

where $\mu=\widehat{\kappa}^{\prime}(0)=\frac{\psi^{\prime}(0)}{-p} \in(0, \infty)$, which finishes the proof.

Remark 4.1. From the Lévy-Khintchine formula, for the case $p, \gamma \in(0, \infty)$,

$$
\widehat{\rho}(s)=\frac{p \sigma^{2}}{2 \gamma}+\frac{p}{\gamma} \int_{0}^{\infty} e^{-s z}\left(\int_{0}^{\infty} e^{-\rho y} \bar{\Pi}(y+z) d y\right) d z .
$$

We are now ready to prove Lemma 2.3 by applying Lemma 2.5 .

Proof of Lemma 2.3. For $y>0$, define the hitting time of $\xi$ by

$$
\tau^{\{y\}}=\inf \left\{t>0, \xi_{t}=y\right\} \text {. }
$$

Since the process $X$ has no positive jumps, $\tau^{\{y\}}=\tau_{y}^{+}+\tau_{y}^{-} \circ \theta_{\tau_{y}^{+}}$. Applying (2.2), (2.6) and Lemma 2.5, we have

$$
\mathbb{P}\left(\tau^{\{y\}}<\infty\right)=\mathbb{E}\left[e^{-p\left(\xi\left(\tau_{y}^{+}\right)-y\right)}\right] \underset{y \rightarrow \infty}{\longrightarrow} \int_{0-}^{\infty} e^{-p z} \rho(d z)=\left(\gamma \Phi^{\prime}(0)\right)^{-1} .
$$

On the other hand, it is proved in Lemma 3.1 of [20] that

$$
\mathbb{P}_{x}\left(\tau^{\{y\}}<\tau_{b}^{+} \wedge \tau_{c}^{-}\right)=\frac{W(b-x)}{W(b-y)}-\frac{W(y-x)}{W(y-c)} \frac{W(b-c)}{W(b-y)},
$$

for $x, y \in(c, b)$. Letting $b \rightarrow \infty$ and $c \rightarrow-\infty$, it follows from (2.5) that

$$
\begin{aligned}
& \mathbb{P}\left(\tau^{\{y\}}<\tau_{c}^{-}\right)=\frac{e^{p c} W(y-c)-W(y)}{W_{p}(y-c)} \text { for } y>c, \\
& \mathbb{P}\left(\tau^{\{y\}}<\infty\right)=e^{p y}-\frac{W(y)}{W_{p}(\infty)}=\frac{1}{\Phi^{\prime}(0)}\left(e^{p y} \Phi^{\prime}(0)-W(y)\right) .
\end{aligned}
$$

Applying (4.4) the proof is completed.

Remark 4.2. For the case of linear Brownian motion with $\psi(s)=\frac{\sigma^{2}}{2} s^{2}-\mu s$ for some constants $\sigma, \mu>0$, we have $\gamma=-\psi^{\prime}(0)=\mu, p=\frac{2 \mu}{\sigma^{2}}$ and $\Phi^{\prime}(0)=\left(\psi^{\prime}(p)\right)^{-1}=\mu^{-1}$. Then $\gamma \Phi^{\prime}(0)=1$. On the other hand, since the Brownian motion has no jumps and due to the positive drift, we always have $\mathbb{P}\left(\tau^{\{y\}}<\infty\right)=1$ for every $y>0$. 


\subsection{Proof of Proposition 2.6}

Since the processes $X$ and $\xi$ are connected via the Lamperti type time transform, in the proofs of our main results, we focus ourself on the study of $\xi$ and its integral functional. In the proof, we write

$$
\vartheta(\cdot):=\frac{1}{R(\cdot)} .
$$

The process $\eta(\cdot)$ in (2.8) is then written as

$$
\eta(t)=\int_{0}^{t} \frac{1}{R\left(\xi_{s}\right)} d s=\int_{0}^{t} \vartheta\left(\xi_{s}\right) d s,
$$

and called the weighted occupation time process in [19], where fluctuation theory of the $\vartheta$-killed spectrally one-sided Lévy processes is studied. In this paper, $\vartheta$ is always assumed to be positive and locally bounded on $(0, \infty)$. Recall that $p$ and $\gamma$ are constants defined in (2.1), respectively, and the Lamperti type identities between the first passage times for $X$ and $\xi$ in (2.11).

For the proof of Proposition 2.6, the condition of explosion is an immediate consequence of the following result from [10]: if $\mathbb{E}\left[\xi_{1}\right] \in(0, \infty)$ and $f$ is a positive locally integrable function, then $\mathbb{P}\left(\int_{0}^{\infty} f\left(\xi_{s}\right) d s<\infty\right) \in\{0,1\}$ and

$$
\mathbb{P}\left(\int_{0}^{\infty} f\left(\xi_{s}\right) d s<\infty\right)=0 \quad \Longleftrightarrow \quad \int^{\infty} f(x) d x=\infty
$$

Even for $\gamma=\infty$, one can find from the proof for sufficiency in [10] that the identity on the right hand side of (4.6) is still a sufficient condition for the left hand side to hold. Therefore, we only focus on the proof of extinction condition in the first statement of Proposition 2.6, where we need the following result that extends the classical result of (2.2) and leave the proof to interested readers; also see Remark 4 in [19] and Lemma 4.2 of [20].

Proposition 4.3. For any $b>x>c>0$, we have

$$
\mathbb{E}_{x}\left[e^{-\eta\left(\tau_{c}^{-}\right)} ; \tau_{c}^{-}<\tau_{b}^{+}\right]=\frac{W^{(\vartheta)}(b, x)}{W^{(\vartheta)}(b, c)}
$$

where $W^{(\vartheta)}$ is defined as the unique locally bounded function satisfying

$$
\begin{aligned}
W^{(\vartheta)}(x, y) & =W(x-y)+\int_{y}^{x} W(x-z) \vartheta(z) W^{(\vartheta)}(z, y) d z \\
& =W(x-y)+\int_{y}^{x} W^{(\vartheta)}(x, z) \vartheta(z) W(z-y) d z .
\end{aligned}
$$

Note that if $\vartheta \equiv q$ for some $q>0$, then $\eta\left(\tau_{c}^{-}\right)=q \tau_{c}^{-}$and $W^{(\vartheta)}(x, y)=W^{(q)}(x-y)$, and the identity in the proposition above exactly reduces to the classical result (2.2). For the $W^{(\vartheta)}(x, y)$ defined above, we have the following asymptotic results.

Lemma 4.4. For any $x, y>0$, we have

$$
\begin{aligned}
W^{(\vartheta)}(x, 0+) & :=\lim _{y \rightarrow 0+} W^{(\vartheta)}(x, y)<\infty \quad \text { if and only if } \int_{0+}^{1} \vartheta(z) W_{p}(z) d z<\infty, \\
\text { and } \quad H^{(\vartheta)}(y) & :=\lim _{x \rightarrow \infty} \frac{W^{(\vartheta)}(x, y)}{W(x)}<\infty \quad \text { if and only if } \quad \int_{1}^{\infty} \vartheta(z) W_{p}(z) d z<\infty .
\end{aligned}
$$


Moreover, the function $H^{(\vartheta)}$ defined above satisfies

$$
\begin{aligned}
H^{(\vartheta)}(y) & =e^{-p y}+\int_{y}^{\infty} e^{-p z} \vartheta(z) W^{(\vartheta)}(z, y) d z \\
& =e^{-p y}+\int_{y}^{\infty} H^{(\vartheta)}(z) \vartheta(z) W(z-y) d z
\end{aligned}
$$

Therefore, if $\vartheta$ satisfies $\mathrm{H}_{1}$, then $H^{(\vartheta)}(0+):=\lim _{y \rightarrow 0+} H^{(\vartheta)}(y)<\infty$.

Proof of Lemma 4.4. We start from the existence of the limits in (4.9).

For $x>y>0$, we have from (4.7) that

$$
\begin{aligned}
\frac{W^{(\vartheta)}(x, y)}{W(x)} & =\frac{W(x-y)}{W(x)}+\int_{y}^{x} \frac{W(x-z)}{W(x)} \vartheta(z) W^{(\vartheta)}(z, y) d z \\
& \leq 1+\int_{y}^{x} e^{-p z} \vartheta(z) W^{(\vartheta)}(z, y) d z
\end{aligned}
$$

Put $G(x):=\exp \left(-\int_{1}^{x} \vartheta(z) W_{p}(z) d z\right)$. Then $G$ is absolutely continuous with respect to Lesbegue measure with $G^{\prime}(x)=-\vartheta(x) W_{p}(x) G(x)$ for a.e. $x$, and for a.e. $x$

$$
\begin{aligned}
& \frac{\partial}{\partial x}\left(G(x)\left(1+\int_{y}^{x} e^{-p z} \vartheta(z) W^{(\vartheta)}(z, y) d z\right)\right) \\
= & G(x)\left(e^{-p x} \vartheta(x) W^{(\vartheta)}(x, y)-\vartheta(x) W_{p}(x)\left(1+\int_{y}^{x} e^{-p z} \vartheta(z) W^{(\vartheta)}(z, y) d z\right)\right) \\
= & G(x) \vartheta(x) W_{p}(x)\left(\frac{W^{(\vartheta)}(x, y)}{W(x)}-\left(1+\int_{y}^{x} e^{-p z} \vartheta(z) W^{(\vartheta)}(z, y) d z\right)\right) \leq 0,
\end{aligned}
$$

by (4.12). Thus, for $x>y>0$

$$
G(x)\left(1+\int_{y}^{x} e^{-p z} \vartheta(z) W^{(\vartheta)}(z, y) d z\right) \leq G(y)
$$

Making use of (4.12) again gives

$$
\frac{W^{(\vartheta)}(x, y)}{W(x)} \leq \frac{G(y)}{G(x)}=\exp \left(\int_{y}^{x} \vartheta(z) W_{p}(z) d z\right) \text { for } x>y>0 .
$$

From the inequality above, if $\int_{1}^{\infty} \vartheta(z) W_{p}(z) d z<\infty$, the function $x \mapsto \frac{W^{(\vartheta)}(x, y)}{W(x)}$ is bounded from above for every fixed $y>0$, which, together with the fact of being increasing in $x$ by (4.11), gives the existence and finiteness of $H^{(\vartheta)}(y)$ on $(0, \infty)$. The equations (4.10) for $H^{(\vartheta)}$ follow from (4.7) and (4.8) by applying the monotone convergence theorem. The "if" part in the first assertion on $W^{(\vartheta)}(x, 0+)$ also follows from the inequality above.

On the other hand, since $W^{(\vartheta)}(x, y) \geq W(x-y)$, we have from (4.8) that,

$$
\begin{aligned}
W^{(\vartheta)}(x, y) & \geq \int_{y}^{x} W(x-z) \vartheta(z) W(z-y) d z \\
& \geq e^{p(x-y)} W_{p}(x-c) \int_{y}^{c} \vartheta(z) W_{p}(z-y) d z \quad \text { for every } c \in(y, x)
\end{aligned}
$$

It follows that, $W^{(\vartheta)}(x, 0+)=\infty$ if $\int_{0+}^{1} \vartheta(z) W_{p}(z) d z=\infty$. Moreover, for every $c>y$,

$$
\liminf _{x \rightarrow \infty} \frac{W^{(\vartheta)}(x, y)}{W(x)} \geq e^{-p y} \int_{y}^{c} \vartheta(z) W_{p}(z-y) d z .
$$


Thus,

$$
\liminf _{x \rightarrow \infty} \frac{W^{(\vartheta)}(x, y)}{W(x)}=\infty \quad \text { if } \quad \int_{1}^{\infty} \vartheta(z) W_{p}(z) d z=\infty,
$$

which proves the "only if" part in the assertions. $H^{(\vartheta)}(0+)<\infty$ under $\mathbb{H}_{1}$ also follows. This completes the proof.

Proof of Proposition 2.6(extinction condition). Letting $c \rightarrow 0+$ in Proposition 4.3, we have

$$
\mathbb{E}_{x}\left[e^{-\eta\left(\tau_{0}^{-}\right)} ; \tau_{0}^{-}<\tau_{b}^{+}\right]=\lim _{c \rightarrow 0+} \frac{W^{(\vartheta)}(b, x)}{W^{(\vartheta)}(b, c)}=\frac{W^{(\vartheta)}(b, x)}{W^{(\vartheta)}(b, 0+)},
$$

for every $b>x>0$. By Lemma 4.4,

$$
\mathbb{P}_{x}\left(\eta\left(\tau_{0}^{-}\right)<\infty, \tau_{0}^{-}<\infty\right)>0 \text { if and only if } \int_{0+}^{1} \vartheta(z) W_{p}(z) d z<\infty .
$$

On the other hand, if $\int_{0+}^{1} \vartheta(z) W_{p}(z) d z<\infty$, we also have for every $q>0$,

$$
\mathbb{E}_{x}\left[e^{-q \cdot \eta\left(\tau_{0}^{-}\right)} ; \tau_{0}^{-}<\tau_{b}^{+}\right]=\frac{W^{(q \vartheta)}(b, x)}{W^{(q \vartheta)}(b, 0)}>0,
$$

where $W^{(q \vartheta)}$ is the generalized scale function in (4.7) with respect to $q \vartheta(\cdot)$. By the scale function identity, for every $x, y, q, r>0$,

$$
W^{(q \vartheta)}(x, y)-W^{(r \vartheta)}(x, y)=(q-r) \int_{y}^{x} W^{(q \vartheta)}(x, z) \vartheta(z) W^{(r \vartheta)}(z, y) d z,
$$

c.f. Lemma 4.3 of [20], we have that $q \rightarrow W^{(q \vartheta)}(x, y)$ is increasing. It is not hard to find that $W^{(q \vartheta)}(x, y) \rightarrow W(x-y)$ as $q \rightarrow 0+$, which shows

$$
\mathbb{P}_{x}\left(\eta\left(\tau_{0}^{-}\right)<\infty, \tau_{0}^{-}<\tau_{b}^{+}\right)=\mathbb{P}_{x}\left(\tau_{0}^{-}<\tau_{b}^{+}\right),
$$

and the second assertion is proved.

Applying Lemma 4.4 to Proposition 4.3 by letting $b \rightarrow \infty$, we also have the the following results on the downward passage time of $X$ and $H^{(\vartheta)}$, and we leave the proof to interested readers. Notice that the result holds for the case that either $p=0$ or $\lim _{z \rightarrow \infty} W_{p}(z)=\Phi^{\prime}(0) \leq \infty$.

Corollary 4.5. Let $H^{(\vartheta)}$ be defined in (4.9) and assume that $\int_{1}^{\infty} \vartheta(z) W_{p}(z) d z<\infty$. Then

$$
\mathbb{E}_{x}\left[e^{-T_{c}^{-}} ; T_{c}^{-}<\infty\right]=\frac{H^{(\vartheta)}(x)}{H^{(\vartheta)}(c)},
$$

for every $x>c>0$. If $R$ satisfies $\mathbb{H}_{1}$, then the identity also holds for $c=0$.

Remark 4.6. If $\int_{1}^{\infty} \vartheta(z) W_{p}(z) d z<\infty$, we can always express $H^{(\vartheta)}$ as a series of integrals from (4.10). On the contrary, if $\int_{1}^{\infty} \vartheta(z) W_{p}(z) d z=\infty$, the Laplace transform in Corollary 4.5 holds for some increasing $\tilde{H}^{(\vartheta)}$ on $(0, \infty)$ satisfying the singular equation

$$
\tilde{H}^{(\vartheta)}(x)=\int_{x}^{\infty} \tilde{H}^{(\vartheta)}(z) \vartheta(z) W(z-x) d z .
$$

To evaluate $H^{(\vartheta)}$, if $\vartheta(x)=\int_{0}^{\infty} e^{-x t} \mu(d t)=\frac{1}{R(x)}$ for some positive measure $\mu$ on $(0, \infty)$ such that the condition $\mathbb{H}_{1}$ is fulfilled, that is,

$$
\int_{0+}^{\infty} \frac{W_{p}(y)}{R(y)} d y=\int_{0+}^{\infty} \vartheta(y) W_{p}(y) d y=\int_{0+}^{\infty} \mu(d t) \int_{0}^{\infty} e^{-y t} W_{p}(y) d y=\int_{0}^{\infty} \frac{\mu(d t)}{\psi_{p}(t)}<\infty,
$$


then $H^{(\vartheta)}(y)=\int_{0}^{\infty} e^{-y s} \nu(d s)$ for some positive measure $\nu$ on $[p, \infty)$ such that

$$
\nu(d s)=\delta_{\{p\}}(d s)+\frac{\nu * \mu(d s)}{\psi(s)} \text { for } s \geq p .
$$

If $\mu(d s)=h(s) d s$ for some measurable $h \geq 0$ on $(0, \infty)$, then $H^{(\vartheta)}(y)=e^{-p y}+\int_{p}^{\infty} e^{-y s} k(s) d s$ where $k$ is a locally integrable function on $(p, \infty)$ satisfying a Volterra equation

$$
k(s)=\frac{1}{\psi(s)}\left(h(s)+\int_{p}^{s} h(s-r) k(r) d r\right) \text { for } s>p .
$$

\subsection{Proof of Theorem 3.1}

An application of Proposition 2.6 shows that, under the condition $p, \gamma \in(0, \infty)$ and $\mathbb{H}_{0}$, $\left\{T_{\infty}^{+}<\infty\right\}=\left\{\tau_{0}^{-}=\infty\right\}$ and has a positive probability, the moment function $m_{n}$ defined in Theorem 3.1 can now be written in terms of $\xi$ as

$$
m_{n}(x)=\mathbb{E}_{x}\left[\eta^{n}(\infty) ; \tau_{0}^{-}=\infty\right] \leq \infty \text { for } n \in \mathbb{N} \text { and } x \geq 0 .
$$

The following proposition on $m_{n}$ is frequently used in our proofs. Similar result can be found in Lemma 8.11.1 of [5], and here we provide a proof for readers' convenience.

Proposition 4.7. Let $U$ be defined in (2.3). We have $m_{0}(x)=1-e^{-p x}$ and

$$
m_{n}(x)=n \int_{0}^{\infty} U(x, d y) \vartheta(y) m_{n-1}(y) \quad \text { for } x \geq 0 \text { and } n \geq 1 .
$$

Proof of Proposition 4.7. The expression for $m_{0}(x)$ follows from (2.2) by taking $q=0$.

Since $\tau_{0}^{-}=t+\tau_{0}^{-} \circ \theta_{t}$ on the set $\left\{t<\tau_{0}^{-}\right\}$for the shifting operator $\theta_{t}$, we have from $d \eta(t)=\vartheta\left(\xi_{t}\right) d t$ that, on the set $\left\{\tau_{0}^{-}=\infty\right\}$,

$$
\begin{aligned}
\eta^{n}(\infty) \cdot \mathbf{1}\left(\tau_{0}^{-}=\infty\right) & =n \int_{0}^{\infty}(\eta(\infty)-\eta(t))^{n-1} \vartheta\left(\xi_{t}\right) \cdot \mathbf{1}\left(t<\tau_{0}^{-}=\infty\right) d t \\
& =n \int_{0}^{\infty}\left(\eta^{n-1}(\infty) \mathbf{1}\left(\tau_{0}^{-}=\infty\right)\right) \circ \theta_{t} \cdot \vartheta\left(\xi_{t}\right) \cdot \mathbf{1}\left(t<\tau_{0}^{-}\right) d t .
\end{aligned}
$$

By the Markov property at time $t>0$ and Fubini's theorem, we complete the proof.

Remark 4.8. If $\gamma \in(0, \infty)$, applying Lemma 2.3 to Proposition 4.7 we have

$$
\mathbb{E}_{x}\left[\eta(\infty) ; \tau_{0}^{-}=\infty\right]<\infty \text { if and only if } \int_{0+}^{\infty} \vartheta(y)\left(1 \wedge\left(y W_{p}(y)\right)\right) d y<\infty .
$$

Using the idea similar to Proposition 4.7 in the following, we have for $x>0$,

$$
\mathbb{E}_{x}\left[\eta\left(\tau_{0}^{-}\right) ; \tau_{0}^{-}<\infty\right]=\int_{0}^{\infty} U(x, d y) \vartheta(y) \mathbb{P}_{y}\left(\tau_{0}^{-}<\infty\right) .
$$

Then Lemma 2.3 shows that if $\gamma \in(0, \infty)$,

$$
\begin{gathered}
\mathbb{E}_{x}\left[\eta\left(\tau_{0}^{-}\right) ; \tau_{0}^{-}<\infty\right]<\infty \text { if and only if } \int_{0+}^{\infty} \vartheta(y)\left(e^{-p y} \wedge W_{p}(y)\right) d y<\infty, \\
\text { and } \mathbb{E}_{x}\left[\eta\left(\tau_{0}^{-}\right)\right]<\infty \text { if and only if } \int_{0+}^{\infty} \vartheta(y) W_{p}(y) d y<\infty .
\end{gathered}
$$

We refer to [23] for more detailed discussions on the related results. Notice that the 0-1 law in the first part of Proposition 2.6 can also be proved by showing that

$$
\mathbb{E}\left[\eta\left(\tau_{0}^{-}\right) ; \tau_{0}^{-}<\tau_{b}^{+}\right]<\infty \text { if and only if } \int_{0+}^{1} \vartheta(z) W_{p}(z) d z<\infty .
$$


We are now ready to prove Theorem 3.1. Notice that $\vartheta=\frac{1}{R}$ in Theorem 3.1 is assumed to satisfy $\mathbb{H}_{1}$, which fulfills the condition of Lemma 4.4, and under which

$$
\eta\left(\tau_{0}^{-}\right)=\eta\left(\tau_{0}^{-}\right) \mathbf{1}\left(\tau_{0}^{-}<\infty\right)+\eta(\infty) \mathbf{1}\left(\tau_{0}^{-}=\infty\right)<\infty \quad \mathbb{P}_{x} \text {-a.s. }
$$

for every $x>0$ as shown in Proposition 2.6.

Proof of Theorem 3.1. The recursive identity (3.1) on the moments of $T_{\infty}^{+}$is obtained from Proposition 4.7. From (4.5), we know that the density of $U$ is bounded by

$$
u(x, y)=e^{-p x} W(y)-W(y-x) \leq W_{p}(y) \quad \text { for all } x, y>0 .
$$

Therefore, with $m_{0}(x)=1-e^{-p x} \leq 1$, we have

$$
m_{n}(x) \leq n \int_{0}^{\infty} \vartheta(y) W_{p}(y) m_{n-1}(y) d y \leq n ! \times\left(\int_{0}^{\infty} \vartheta(y) W_{p}(y)\right)^{n} \quad \text { for all } n \geq 1 .
$$

Since Carleman's condition on the moments is satisfied, the distribution of $T_{\infty}^{+}=\eta(\infty)$ on the set $\left\{T_{\infty}^{+}<T_{0}^{-}\right\}=\left\{\tau_{0}^{-}=\infty\right\}=\left\{T_{0}^{-}=\infty\right\}=\left\{T_{\infty}^{+}<\infty\right\}$ under the condition $\mathbb{H}_{1}$ is uniquely determined by its moments $\left(m_{n}\right)_{n \geq 0}$, and the desired conclusion follows.

\subsection{Proofs of Theorems 3.3 and 3.6}

To compare the asymptotic behaviors of functions at infinity, we write as usual

$$
f(x) \sim g(x) \quad \text { as } \quad x \rightarrow \infty \quad \text { if } \quad \lim _{x \rightarrow \infty} f(x) / g(x)=1,
$$

and

$$
f(x)=o(g(x)) \quad \text { as } \quad x \rightarrow \infty \quad \text { if } \quad \lim _{x \rightarrow \infty} f(x) / g(x)=0,
$$

where $g(x) \neq 0$ for $x$ large enough. We always assume that $p, \gamma \in(0, \infty)$ and the weight function $\vartheta$ satisfies $\mathbb{H}_{0}$ and $\mathbb{H}_{2}$. We prove in Proposition 4.10 the asymptotic results about the tail integrals for functions of this kind, that is,

$$
\int_{x+y}^{\infty} f(z) d z / \int_{y}^{\infty} f(z) d z \rightarrow \exp (-\lambda x) \quad \text { as } y \rightarrow \infty,
$$

for some constant $\lambda \in[0, \infty)$ and every $x>0$. Since the condition (4.13) is closely related to regularly varying functions as shown in Remark 3.2, similar results for "Stieltjesintegral forms" can be found in Theorem 1.6.4 and 1.6.5 of [5]. Recall the following Karamata's theorem from Theorem 1.5.11 of [5].

Proposition 4.9 (Karamata's Theorem). Let $f$ vary regularly with index $\rho$, and be locally bounded in $[c, \infty)$. Then

(i) for any $\sigma \geq-(\rho+1)$,

$$
x^{\sigma+1} f(x) / \int_{c}^{x} t^{\sigma} f(t) d t \rightarrow \sigma+\rho+1 \text { as } x \rightarrow \infty ;
$$

(ii) for any $\sigma<-(\rho+1)$ (and for $\sigma=-(\rho+1)$ if $\int^{\infty} t^{-(\rho+1)} f(t) d t<\infty$ ),

$$
x^{\sigma+1} f(x) / \int_{x}^{\infty} t^{\sigma} f(t) d t \rightarrow-(\sigma+\rho+1) \text { as } x \rightarrow \infty .
$$

Proposition 4.10. Suppose that a positive function $f$ has a finite tail integral and its tail integral satisfies the condition (4.13) for some $\lambda \in[0, \infty)$, 
(A) If $\lambda=0$, then for any $\alpha>0$ we have

$$
\int_{1}^{\infty} e^{\alpha y} f(y) d y=\infty, \quad \int_{1}^{\infty} e^{\alpha y} \int_{y}^{\infty} f(z) d z d y=\infty
$$

and

$$
e^{-\alpha x} \int_{1}^{x} e^{\alpha y} f(y) d y=o\left(\int_{x}^{\infty} f(y) d y\right) \quad \text { as } x \rightarrow \infty .
$$

(B) If $\lambda>0$, then for any $\alpha<\lambda$ we have

$$
\int_{1}^{\infty} e^{\alpha y} f(y) d y<\infty, \quad \int_{1}^{\infty} e^{\alpha y} \int_{y}^{\infty} f(z) d z d y<\infty,
$$

and as $x \rightarrow \infty$,

$$
\int_{x}^{\infty} e^{\alpha(y-x)} \int_{y}^{\infty} f(z) d z d y \sim \lambda^{-1} \int_{x}^{\infty} e^{\alpha(y-x)} f(y) d y \sim \frac{1}{\lambda-\alpha} \int_{x}^{\infty} f(y) d y .
$$

(C) If $\lambda>0$, denoting by $k$ the inverse of function $x \rightarrow \int_{x}^{\infty} f(y) d y$, i.e. $\int_{k(x)}^{\infty} f(y) d y=x$ for all small $x>0$, we have

$$
k(x) \sim-\lambda^{-1} \log x \quad \text { as } x \rightarrow 0+.
$$

Proof of Proposition 4.10. Put $g(u):=\int_{\log u}^{\infty} f(z) d z$. It is true that $u^{\lambda} g(u)$ is slowly varying under the condition (4.13). In the following discussion, we take $u=e^{x}$.

For $\alpha \in \mathbb{R}$ and $x>1$, by change of variable and Fubini's theorem, we have

$$
\begin{aligned}
\int_{x}^{\infty}\left(e^{\alpha y}-e^{\alpha x}\right) f(y) d y=\alpha \int_{x}^{\infty} \int_{x}^{y} e^{\alpha z} f(y) d z d y \\
=\alpha \int_{x}^{\infty} e^{\alpha z} \int_{z}^{\infty} f(y) d y d z=\alpha \int_{u}^{\infty} y^{\alpha-1} g(y) d y .
\end{aligned}
$$

Applying Proposition 1.5.1 of [5], the last integral converges if $\alpha-\lambda<0$ and diverges if $\alpha-\lambda>0$, which proves those results on the finiteness of integrals $\int_{1}^{\infty} e^{\alpha y} \int_{y}^{\infty} f(z) d z d y$ and $\int_{1}^{\infty} e^{\alpha y} f(y) d y$ in (A) and (B), respectively.

If $\lambda>0$ and $\alpha-\lambda<0$, applying Karamata's theorem, we further have

$$
\alpha \int_{u}^{\infty} y^{\alpha-1} g(y) d y \sim \frac{\alpha}{\lambda-\alpha}\left(u^{\alpha} g(u)\right)=\frac{\alpha}{\lambda-\alpha} e^{\alpha x} \int_{x}^{\infty} f(y) d y,
$$

which proves the last result of (B).

If $\lambda=0$ and $\alpha>0$, then $u^{\alpha} g(u) \rightarrow \infty$ as $u \rightarrow \infty$. By integration by parts, we have

$$
\begin{aligned}
\int_{1}^{x} e^{\alpha y} f(y) d y & =e^{\alpha} \int_{1}^{\infty} f(y) d y-e^{\alpha x} \int_{x}^{\infty} f(y) d y+\alpha \int_{1}^{x} e^{\alpha y} d y \int_{y}^{\infty} f(z) d z \\
& =e^{\alpha} g(e)-\left(u^{\alpha} g(u)-\alpha \int_{e}^{u} z^{\alpha-1} g(z) d z\right) .
\end{aligned}
$$

Since $u^{\alpha} g(u) \sim \alpha \int_{e}^{u} z^{\alpha-1} g(z) d z$ by Karamata's theorem, the last result of (A) holds.

If $\lambda>0$, for any $\varepsilon>0$, by Proposition 1.5.1 of [5], we have $u^{\lambda+\varepsilon} g(u) \rightarrow \infty$ and $u^{\lambda-\varepsilon} g(u) \rightarrow 0+$ as $u \rightarrow \infty$. Since $g(u)$ is continuous and decreasing, for any fixed $M>0$ we have $g\left(e^{k(x)}\right)=x$ and

$$
e^{(\lambda-\varepsilon) k(x)} x \leq M^{-1} \text { and } e^{(\lambda+\varepsilon) k(x)} x \geq M,
$$

for all small enough $x>0$. Therefore,

$$
\frac{-1}{\lambda+\varepsilon}(\log x-\log M) \leq k(x) \leq \frac{-1}{\lambda-\varepsilon}(\log x+\log M),
$$

which leads to the result of (C). 
Applying Lemma 2.3 and Proposition 4.10 above, we first obtain the following asymptotic result on the integral with respect to potential measure $U$ of function $f$ which satisfies (4.13) with $\lambda=0$.

Lemma 4.11. Suppose that $\gamma \in(0, \infty)$ and $f \geq 0$ is an integrable function on $(0, \infty)$ with

$$
\int_{x+a}^{\infty} f(y) d y \sim \int_{x}^{\infty} f(y) d y \text { for every } a>0 .
$$

Then

$$
\int_{0}^{\infty} f(y) U(x, d y) \sim \gamma^{-1} \int_{x}^{\infty} f(y) d y \quad \text { as } x \rightarrow \infty .
$$

Proof of Lemma 4.11. Notice that $W(y-x)=0$ for $y<x$, we have from (2.3) that for $x>0$,

$$
\begin{aligned}
& \int_{0}^{\infty} f(y) u(x, y) d y \\
= & \int_{0}^{\infty}\left(e^{-p x} W(y)-W(y-x)\right) f(y) d y \\
= & e^{-p x} \int_{0}^{x} W(y) f(y) d y+\int_{0}^{\infty}\left(e^{-p x} W(x+y)-W(y)\right) f(x+y) d y \\
= & : I_{1}+I_{2} .
\end{aligned}
$$

Since $W(y) e^{-p y}=W_{p}(y) \uparrow \Phi^{\prime}(0)<\infty$ as $y \rightarrow \infty$, we have from Proposition 4.10(A) that

$$
I_{1} \leq \Phi^{\prime}(0) e^{-p x} \int_{0}^{x} e^{p y} f(y) d y=o\left(\int_{x}^{\infty} f(y) d y\right) .
$$

On the other hand, for every $\varepsilon>0$, applying Lemma 2.3, for some $k>0$,

$$
\left|\left(e^{-p x} W(x+y)-W(y)\right)-\gamma^{-1}\right| \leq \varepsilon \gamma^{-1}, \quad \text { for } x, y>k .
$$

Since $e^{-p x} W(x+y)-W(y) \leq \Phi^{\prime}(0)$ by (4.5), then for $x>k$

$$
\begin{aligned}
\mid I_{2}-\gamma^{-1} & \int_{x}^{\infty} f(y) d y \mid \\
& \leq\left(\Phi^{\prime}(0)+\gamma^{-1}\right) \int_{0}^{k} f(x+y) d y+\varepsilon \gamma^{-1} \int_{k}^{\infty} f(x+y) d y \\
& =\left(\Phi^{\prime}(0)+\gamma^{-1}-\varepsilon \gamma^{-1}\right)\left(\int_{x}^{\infty} f(y) d y-\int_{x+k}^{\infty} f(y) d y\right)+\varepsilon \gamma^{-1} \int_{x}^{\infty} f(y) d y \\
& \sim \varepsilon \gamma^{-1} \cdot \int_{x}^{\infty} f(y) d y \text { as } x \rightarrow \infty,
\end{aligned}
$$

where we used the assumption that $\int_{x}^{\infty} f(y) d y \sim \int_{x+k}^{\infty} f(y) d y$ for $k>0$ as $x \rightarrow \infty$. This finishes the proof.

We are now ready to prove part (A) of Theorem 3.3. Denote

$$
J\left(\tau_{x}^{+}\right):=\int_{\tau_{x}^{+}}^{\infty} \vartheta\left(\xi_{t}\right) d t=T_{\infty}-T_{x}^{+} \quad \text { on the set }\left\{\tau_{0}^{-}=\infty\right\} .
$$

We start with investigating the asymptotic behaviors of the first two moments of $\eta(\infty)$ under $\mathbb{P}_{x}$, and then estimate the first two moments of $J\left(\tau_{z}^{+}\right)$under $\mathbb{P}_{x}$ using the Markov property of $\xi$. Recall that $\varphi$ is the tail integral defined in (2.13) and $p, \gamma \in(0, \infty)$ in (2.1).

We now present the proof for Theorem 3.3(A). The proof for Theorem 3.3(B) is deferred. 
Proof of Theorem 3.3(A). In this case the tail integral function $\varphi$ of $\vartheta$ is assumed to satisfy condition (4.13) with $\lambda=0$ and $p, \gamma \in(0, \infty)$.

We first show that for any $x>0$,

$$
\lim _{z \rightarrow \infty} \mathbb{E}_{x}^{\uparrow}\left[\left(\frac{J\left(\tau_{z}^{+}\right)}{\varphi(z)}-1\right)^{2}\right]=0,
$$

under a stronger condition

$$
\varphi(0)=\frac{1}{\gamma} \int_{0}^{\infty} \vartheta(y) d y<\infty,
$$

which holds under condition $\mathbb{H}_{1}$ and ensures $m_{2}(x)<\infty$ by Theorem 3.1, and implies the desired convergence in probability in Theorem 3.3 (A). If $\vartheta$ fails to be integrable at 0 , we can apply (4.14) to the function $\vartheta_{c}(\cdot)=\vartheta(\cdot+c)$ under $\mathbb{P}_{x-c}^{\uparrow}$ for every $x>c>0$. Note that the associated tail integral function for $\vartheta_{c}$ is $\varphi_{c}(x)=\varphi(x+c)$. We then have

$$
\begin{aligned}
& \mathbb{P}_{x-c}\left(\left|\frac{1}{\varphi_{c}(z-c)} \int_{\tau_{z-c}^{+}}^{\infty} \vartheta_{c}\left(\xi_{t}\right) d t-1\right| \geq \varepsilon \mid \tau_{0}^{-}=\infty\right) \\
= & \mathbb{P}_{x}\left(\left|\frac{J\left(\tau_{z}^{+}\right)}{\varphi(z)}-1\right| \geq \varepsilon \mid \tau_{c}^{-}=\infty\right) \rightarrow 0 \quad \text { as } z \rightarrow \infty,
\end{aligned}
$$

where we need the spatial homogeneousness of $\xi$ for the identity. Letting $c \rightarrow 0+$ and noticing that $p>0$, the desired convergence in probability in Theorem 3.3 (A) also holds.

Recall the moments $m_{1}$ and $m_{2}$ in Proposition 4.7,

$$
\begin{aligned}
& m_{1}(z)=\int_{0}^{\infty} u(z, y) \vartheta(y)\left(1-e^{-p y}\right) d y, \\
& m_{2}(z)=2 \int_{0}^{\infty} u(z, y) \vartheta(y) m_{1}(y) d y .
\end{aligned}
$$

We claim that, for every $x>0$, as $z \rightarrow \infty$,

$$
m_{1}(z) \sim \varphi(z), \quad m_{2}(z) \sim \varphi^{2}(z),
$$

and in addition,

$$
\mathbb{E}_{x}\left[h\left(\xi\left(\tau_{z}^{+}\right)\right) ; \tau_{z}^{+}<\tau_{0}^{-}\right] \sim \mathbb{P}_{x}\left(\tau_{0}^{-}=\infty\right) g(z),
$$

where $h$ and $g$ are functions such that $h \sim g$ and $g$ is a deceasing function satisfying $g(z+a) \sim g(z)$ for every $a \in \mathbb{R}$.

Actually, given (4.17) and (4.18), since $J\left(\tau_{z}^{+}\right)=\eta(\infty) \circ \theta_{\tau_{z}^{+}}$, we have for $z>x>0$,

$$
\begin{aligned}
\mathbb{E}_{x}^{\uparrow}\left[J\left(\tau_{z}^{+}\right)\right] & =\frac{1}{\mathbb{P}_{x}\left(\tau_{0}^{-}=\infty\right)} \mathbb{E}_{x}\left[m_{1}\left(\xi\left(\tau_{z}^{+}\right)\right) ; \tau_{z}^{+}<\tau_{0}^{-}\right] \sim \varphi(z), \\
\mathbb{E}_{x}^{\uparrow}\left[J^{2}\left(\tau_{z}^{+}\right)\right] & =\frac{1}{\mathbb{P}_{x}\left(\tau_{0}^{-}=\infty\right)} \mathbb{E}_{x}\left[m_{2}\left(\xi\left(\tau_{z}^{+}\right)\right) ; \tau_{z}^{+}<\tau_{0}^{-}\right] \sim \varphi^{2}(z),
\end{aligned}
$$

which implies the $L^{2}$ convergence with respect to $\mathbb{P}_{x}^{\uparrow}$ in (4.14).

To prove (4.17), we apply Lemma 4.11 to the function $f_{1}(y)=\vartheta(y)\left(1-e^{-p y}\right)$. It is not hard to see that as $z \rightarrow \infty$

$$
\int_{z}^{\infty} f_{1}(y) d y \sim \int_{z}^{\infty} \vartheta(y) d y \sim \int_{z+a}^{\infty} \vartheta(y) d y \sim \int_{z+a}^{\infty} f_{1}(y) d y .
$$

Thus, $f_{1}$ fulfills the condition in Lemma 4.11. It follows from (4.15) that

$$
m_{1}(z)=\int_{0}^{\infty} u(z, y) f_{1}(y) d y \sim \gamma^{-1} \int_{z}^{\infty} f_{1}(y) d y \sim \varphi(z) \quad \text { as } z \rightarrow \infty .
$$


Then, we take $f_{2}(y)=\vartheta(y) m_{1}(y)$. From the result above, for any $\varepsilon \in(0,1)$

$$
(1-\varepsilon) \varphi(z) \leq m_{1}(z) \leq(1+\varepsilon) \varphi(z) \text { for all } z>k_{1},
$$

for some $k_{1}>0$. It follows that for $z>k_{1}$

$$
\int_{z}^{\infty} f_{2}(y) d y \leq(1+\varepsilon) \int_{z}^{\infty} \vartheta(y) \varphi(y) d y=\frac{1+\varepsilon}{2} \gamma \varphi^{2}(z),
$$

and

$$
\int_{z}^{\infty} f_{2}(y) d y \geq(1-\varepsilon) \int_{z}^{\infty} \vartheta(y) \varphi(y) d y=\frac{1-\varepsilon}{2} \gamma \varphi^{2}(z),
$$

which gives that

$$
\int_{z}^{\infty} f_{2}(y) d y \sim \frac{1}{2} \gamma \varphi^{2}(z)
$$

and $f_{2}$ satisfies the condition of Lemma 4.11. Applying (4.16) and Lemma 4.11 we have

$$
m_{2}(z)=2 \int_{0}^{\infty} f_{2}(y) u(z, y) d y \sim 2 \gamma^{-1} \int_{z}^{\infty} f_{2}(y) d y \sim \varphi^{2}(z) \quad \text { as } z \rightarrow \infty .
$$

To prove (4.18), let $k_{2}>0$ satisfy

$$
\rho\left(\left[0, k_{2}\right]\right) \geq 1-\varepsilon \text { and }(1-\varepsilon) g(z) \leq h(z) \leq(1+\varepsilon) g(z) \text { for } z>k_{2},
$$

where $\rho$ is the stationary overshoot distribution in (2.7). Then for $z>k_{2}$,

$$
\begin{aligned}
(1+\varepsilon) & \geq \frac{\mathbb{E}\left[h\left(\xi_{\tau_{z}^{+}}\right)\right]}{g(z)} \geq(1-\varepsilon) \frac{\mathbb{E}\left[g\left(\xi_{\tau_{z}^{+}}\right)\right]}{g(z)} \\
& \geq(1-\varepsilon) \frac{\left.\mathbb{E}\left[g\left(\xi_{\tau_{z}^{+}}\right) ; \xi_{\tau_{z}^{+}}<z+k_{2}\right)\right]}{g(x)} \geq(1-\varepsilon)^{2} \frac{g\left(z+k_{2}\right)}{g(z)},
\end{aligned}
$$

where the monotonicity of $g$ is applied to the first and the last inequality. Thus,

$$
\mathbb{E}\left[h\left(\xi_{\tau_{z}^{+}}\right)\right] \sim g(z) \quad \text { as } z \rightarrow \infty .
$$

Lastly, applying the strong Markov property for $\xi$ we further have

$$
\begin{aligned}
& \mathbb{E}_{x}\left[h\left(\xi_{\tau_{z}^{+}}\right) ; \tau_{z}^{+}<\tau_{0}^{-}\right] \\
= & \mathbb{E}_{x}\left[h\left(\xi_{\tau_{z}^{+}}\right)\right]-\mathbb{P}_{x}\left(\tau_{0}^{-}<\tau_{z}^{+}\right) \cdot \mathbb{E}\left[h\left(\xi_{\tau_{z}^{+}}\right)\right] \\
= & \mathbb{E}\left[h\left(\xi_{\tau_{z-x}^{+}}+x\right)\right]-\mathbb{E}\left[h\left(\xi_{\tau_{z}^{+}}\right)\right]+\mathbb{P}_{x}\left(\tau_{z}^{+}<\tau_{0}^{-}\right) \cdot \mathbb{E}\left[h\left(\xi_{\tau_{z}^{+}}\right)\right] \\
\sim & \mathbb{P}_{x}\left(\tau_{z}^{+}<\tau_{0}^{-}\right) \cdot g(z) \sim \mathbb{P}_{x}\left(\tau_{0}^{-}=\infty\right) g(z) \text { as } z \rightarrow \infty .
\end{aligned}
$$

This finishes the proof.

Remark 4.12. In the proof of statement (A), we have

$$
\mathbb{E}_{x}^{\uparrow}\left[J\left(\tau_{z}^{+}\right)\right] \sim \mathbb{E}_{z}^{\uparrow}[\eta(\infty)] \sim \varphi(z) \text { as } z \rightarrow \infty .
$$

However, with the presence of positive jumps, $m_{1}(x)$ and $\mathbb{E}_{x}^{\uparrow}[\eta(\infty)]$ may fail to be monotone in $x$ in general.

For the proof of statement (B) of Theorem 3.3, we make use of the local time for the process $\xi$, see c.f. Chapter V of [2] for more detailed discussion. Given a SPLP $\xi$, its local time exists and is defined as the density of occupation measure by,

$$
L(y, t):=\lim _{\varepsilon \rightarrow 0+} \frac{1}{2 \varepsilon} \int_{0}^{t} \mathbf{1}\left(\left|\xi_{s}-y\right|<\varepsilon\right) d s, \quad \text { for } y \in \mathbb{R}, t>0, \mathbb{P} \text {-a.s. }
$$


and the following occupation density formula holds for all bounded Borel function $f \geq 0$,

$$
\int_{0}^{t} f\left(\xi_{s}\right) d s=\int_{-\infty}^{\infty} f(y) L(y, t) d y \quad \mathbb{P} \text {-a.s.. }
$$

We also need the following Lemmas on the regularly varying functions and the local time.

Lemma 4.13. Let $\vartheta$ be the function in statement (B) of Theorem 3.3. Let $f \geq 0$ be a measurable function locally integrable such that the set $\{x \in \mathbb{R}, f(x)>0\}$ is bounded from below,

$$
\int_{-\infty}^{\infty} e^{-\lambda y} f(y) d y<\infty \text { and } \int_{-\infty}^{\infty} e^{-2 \alpha y} f^{2}(y) d y<\infty \text { for some } 2 \alpha \in(0, \lambda) .
$$

Then

$$
\frac{1}{\varphi(x)} \int_{-\infty}^{\infty} \vartheta(x+y) f(y) d y \underset{x \rightarrow \infty}{\longrightarrow} \lambda \gamma \int_{-\infty}^{\infty} e^{-\lambda y} f(y) d y
$$

Lemma 4.14. Suppose that $p>0$. For any $2 \alpha \in(0, p)$, we have

$$
\mathbb{E}\left[\int_{-\infty}^{\infty} e^{-2 \alpha y} L^{2}(y, \infty) d y\right]<\infty .
$$

Remark 4.15. Lemma 4.13 appears similar to the Abelian theorem, c.f. Theorem 4.1.3 of [5] where $\vartheta(\log \cdot)$ is assumed to be regularly varying, and also similar to Theorem 1.7.5 in [5], where conditions related to slowly decreasing is imposed. The condition here can be replaced by other, possibly weaker, conditions. For example, if $f$ has bounded variation and is bounded, right-continuous, and $\{x \in \mathbb{R}, f(x)>0\}$ is bounded from below, an application of the uniform convergence theorem could give the same result.

Proof of Lemma 4.13. Since the set $\{x \in \mathbb{R}, f(x)>0\}$ is bounded from below, it is sufficient to prove (4.21) for $f$ vanishing on $(-\infty, 0)$, and we only focus on integrals on $(0, \infty)$.

By the assumption in statement (B), for some $K, M>0$, we have

$$
\int_{x}^{\infty} \vartheta^{2}(y) d y \leq M \cdot \varphi^{2}(x) \text { for all } x>K .
$$

Applying Fubini's theorem, for $x>K$ we have

$$
\begin{aligned}
& \int_{0}^{\infty}\left(e^{2 \alpha y}-1\right) \vartheta^{2}(x+y) d y=\int_{0}^{\infty} \vartheta^{2}(x+y) d y \int_{0}^{y} 2 \alpha e^{2 \alpha t} d t \\
= & 2 \alpha \int_{0}^{\infty} e^{2 \alpha t} d t \int_{x+t}^{\infty} \vartheta^{2}(y) d y \leq 2 \alpha M \int_{0}^{\infty} e^{2 \alpha t} \varphi^{2}(x+t) d t \\
= & 2 \alpha M \int_{x}^{\infty} e^{2 \alpha(t-x)} \varphi^{2}(t) d t=\left.2 \alpha M\left(u^{-2 \alpha} \int_{u}^{\infty} s^{2 \alpha-1} \varphi^{2}(\log s) d s\right)\right|_{u=e^{x}} \\
\sim & \left.\frac{\alpha \cdot M}{\lambda-\alpha} \varphi^{2}(\log u)\right|_{u=e^{x}}=\frac{\alpha \cdot M}{\lambda-\alpha} \cdot \varphi^{2}(x) \text { as } x \rightarrow \infty,
\end{aligned}
$$

by applying Karamata's theorem to the last line since $s \rightarrow \varphi^{2}(\log s)$ is regularly varying with index $-2 \lambda$. Thus, under the assumption for Theorem 3.3 (B),

$$
\limsup _{x \rightarrow \infty} \int_{0}^{\infty} e^{2 \alpha y} \frac{\vartheta^{2}(x+y)}{\varphi^{2}(x)} d y<\infty .
$$

The Cauchy-Schwarz inequality then yields

$$
\left(\int_{0}^{\infty} \frac{\vartheta(x+y)}{\varphi(x)} f(y) d y\right)^{2} \leq\left(\int_{0}^{\infty} e^{-2 \alpha y} f^{2}(y) d y\right) \cdot\left(\int_{0}^{\infty} e^{2 \alpha y} \frac{\vartheta^{2}(x+y)}{\varphi^{2}(x)} d y\right),
$$


where the second term on the right hand side is dominated by some constant.

On the other hand, it is not hard to check that the limit (4.21) holds for simple functions $f(x)=\mathbf{1}(x>c), \forall c \geq 0$ as well as their linear combinations. Moreover, it holds for any bounded measurable function $f$ on $(0, \infty)$ which can be uniformly and non-decreasingly approximated by simple functions $f_{n}$ satisfying (4.21). Therefore, it holds for all nonnegative bounded Borel functions on $(0, \infty)$, by applying the functional monotone convergence theorem, c.f. Theorem 2.12.9 [6]. Finally, for any function $f$ satisfying the assumption of Lemma 4.13, taking $f_{n}=f \wedge n$ and applying (4.22) gives

$$
\lim _{n \rightarrow \infty} \limsup _{x \rightarrow \infty}\left(\int_{0}^{\infty} \frac{\vartheta(x+y)}{\varphi(x)}\left(f(y)-f_{n}(y)\right) d y\right)^{2}=0 .
$$

It follows that

$$
\begin{gathered}
\lim _{x \rightarrow \infty} \int_{0}^{\infty} \frac{\vartheta(x+y)}{\varphi(x)} f(y) d y=\lim _{n \rightarrow \infty} \lim _{x \rightarrow \infty} \int_{0}^{\infty} \frac{\vartheta(x+y)}{\varphi(x)} f_{n}(y) d y \\
=\lim _{n \rightarrow \infty} \lambda \gamma \int_{0}^{\infty} e^{-\lambda y} f_{n}(y) d y=\lambda \gamma \int_{0}^{\infty} e^{-\lambda y} f(y) d y .
\end{gathered}
$$

This finishes the proof.

Lemma 4.14 is proved following the argument used in Theorem V.1 of [2], where Plancherel's theorem is applied.

Proof of Lemma 4.14. Let $2 \alpha \in(0, p)$ and $g(y)=e^{-\alpha y} L(y, \infty)$ for $y \in \mathbb{R}$. Then $\psi(\alpha), \psi(2 \alpha)$ $<0$ by definition. Applying Fubini's theorem and the occupation density formula, we have

and

$$
\mathbb{E}\left[\int_{0}^{\infty} e^{-2 \alpha \xi_{t}} d t\right]=\int_{0}^{\infty} \mathbb{E}\left[e^{-2 \alpha \xi_{t}}\right] d t=\frac{-1}{\psi(2 \alpha)},
$$

$$
\mathbb{E}\left[\int_{-\infty}^{\infty} e^{-\alpha y} L(y, \infty) d y\right]=\mathbb{E}\left[\int_{0}^{\infty} e^{-\alpha \xi_{t}} d t\right]=\frac{-1}{\psi(\alpha)} .
$$

Thus, $e^{-\alpha \xi_{t}}, e^{-2 \alpha \xi_{t}}$ and $g(y)$ are all integrable. The Fourier transform of $g$ gives for every $u \in \mathbb{R}$,

$$
\mathcal{F} g(u):=\int_{-\infty}^{\infty} e^{i u y} e^{-\alpha y} L(y, \infty) d y=\int_{0}^{\infty} e^{(i u-\alpha) \xi_{t}} d t, \quad \mathbb{P} \text {-a.s.. }
$$

In addition, we have

$$
\begin{aligned}
\mathbb{E}\left[|\mathcal{F} g(u)|^{2}\right] & =\mathbb{E}[\mathcal{F} g(u) \mathcal{F} g(-u)]=\mathbb{E}\left[\int_{0}^{\infty} \int_{0}^{\infty} e^{(i u-\alpha) \xi_{t}+(-i u-\alpha) \xi_{s}} d t d s\right] \\
& =\mathbb{E}\left[\int_{0}^{\infty} \int_{0}^{\infty} e^{(i u-\alpha)\left(\xi_{t}-\xi_{s}\right)-2 \alpha \xi_{s}} d t d s\right] .
\end{aligned}
$$

Given the integrability of $e^{-\alpha \xi_{t}}$ and $e^{-2 \alpha \xi_{t}}$, we can re-express the last term as

$$
2 \Re\left(\int_{0}^{\infty} d s \int_{s}^{\infty} d t \mathbb{E}\left[e^{(i u-\alpha)\left(\xi_{t}-\xi_{s}\right)}\right] \mathbb{E}\left[e^{-2 \alpha \xi_{s}}\right]\right) .
$$

Recall the measure $\mathbb{P}^{(\alpha)}$ defined in (2.4). Under the new measure $\mathbb{P}^{(\alpha)}$ the above quantity equals to

$$
2 \Re\left(\int_{0}^{\infty} e^{\psi(\alpha) t} \mathbb{E}\left[e^{i u \xi_{t}} e^{-\alpha \xi_{t}-\psi(\alpha) t}\right] d t \int_{0}^{\infty} \mathbb{E}\left[e^{-2 \alpha \xi_{s}}\right] d s\right)=\frac{-2}{\psi(2 \alpha)} \Re\left(\frac{1}{\Psi_{\alpha}(u)-\psi(\alpha)}\right),
$$

where

$$
\Psi_{\alpha}(s)=t^{-1} \log \mathbb{E}^{(\alpha)}\left[e^{i s \xi_{t}}\right]=-\psi_{\alpha}(-i s)
$$


is the characteristic exponent of $\xi$ under $\mathbb{P}^{(\alpha)}$. Noticing that $-\psi(\alpha)>0$, we have

$$
\int_{-\infty}^{\infty} \mathbb{E}\left[|\mathcal{F} g(u)|^{2}\right] d u=\frac{-2}{\psi(2 \alpha)} \int_{-\infty}^{\infty} \Re\left(\frac{1}{\Psi_{\alpha}(u)-\psi(\alpha)}\right) d u<\infty
$$

where Theorem II.16 in [2] is applied. The proof is finished by applying Plancherel's theorem.

We are now ready to prove the result of part (B).

Proof of Theorem 3.3(B). Recall that $p, \gamma \in(0, \infty)$. Let $\lambda>0$ be the constant in condition $\mathrm{H}_{2}$, and $f, g$ be bounded continuous and nonnegative functions.

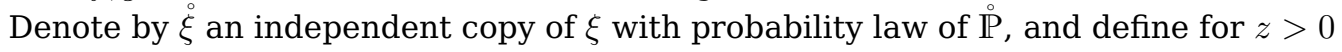

$$
G(z):=\stackrel{\circ}{\mathbb{E}}\left[g\left(\int_{0}^{\infty} \frac{\vartheta\left(\stackrel{\circ}{\xi}_{t}+z\right)}{\varphi(z)} d t\right) \mid \stackrel{\circ}{\tau}-_{-z}^{-}=\infty\right] .
$$

Applying the strong Markov property of $\xi$ at $\tau_{x}^{+}$, we have

$$
\mathbb{E}_{x}^{\uparrow}\left[f\left(\frac{\varphi\left(\xi\left(\tau_{z}^{+}\right)\right)}{\varphi(z)}\right) \cdot g\left(\frac{J\left(\tau_{z}^{+}\right)}{\varphi\left(\xi\left(\tau_{z}^{+}\right)\right)}\right)\right]=\mathbb{E}_{x}^{\uparrow}\left[f\left(\frac{\varphi\left(\xi\left(\tau_{z}^{+}\right)\right)}{\varphi(z)}\right) \cdot G\left(\xi\left(\tau_{z}^{+}\right)\right)\right] .
$$

Let $\stackrel{\circ}{L}(y, t)$ be the local time of $\stackrel{\circ}{\xi}$ at level $y$ and time $t$. Since $\dot{\xi}(t) \rightarrow \infty$, we have from (4.6) that $\int_{0}^{\infty} e^{-\lambda \dot{\xi}_{t}} d t<\infty \stackrel{\circ}{\mathrm{P}}$-a.s.. Applying Theorem I.20 of [2], Lemma 4.14 and the fact that $\left|\inf _{t>0} \dot{\xi}_{t}\right|<\infty$, one can check that, for $2 \alpha<\lambda \wedge p, \stackrel{\circ}{L}(y, \infty)$ fulfills the conditions of Lemma $4.13 \stackrel{\circ}{\mathbb{P}}$-a.s. Therefore,

$$
\begin{gathered}
\int_{0}^{\infty} \frac{\vartheta\left(\stackrel{\circ}{\xi}_{t}+z\right)}{\varphi(z)} d t=\int_{-\infty}^{\infty} \frac{\vartheta(z+y) \stackrel{\circ}{L}(y, \infty)}{\varphi(z)} d y \\
\underset{z \rightarrow \infty}{\longrightarrow} \lambda \gamma \int_{-\infty}^{\infty} e^{-\lambda y} \stackrel{\circ}{L}(y, \infty) d y=\lambda \gamma \int_{0}^{\infty} e^{-\lambda \dot{\xi}_{t}} d t, \quad \stackrel{\circ}{\mathbb{P}} \text {-a.s.. }
\end{gathered}
$$

Moreover, since $\stackrel{\circ}{\mathbb{P}}\left(\lim _{z \rightarrow+\infty} \mathbf{1}\left({\stackrel{\circ}{\tau_{-z}^{-}}}^{-}=\infty\right)=1\right)=1$, by the dominated convergence theorem

$$
G(z)=\frac{1}{1-e^{-p z}} \stackrel{\circ}{\mathbb{E}}\left[g\left(\int_{0}^{\infty} \frac{\vartheta\left(\stackrel{\circ}{\xi}_{t}+z\right)}{\varphi(z)} d t\right) \cdot \mathbf{1}\left(\stackrel{\circ}{\tau}-z^{-}=\infty\right)\right] \underset{z \rightarrow \infty}{\longrightarrow} \stackrel{\circ}{\mathbb{E}}\left[g\left(\lambda \gamma \int_{0}^{\infty} e^{-\lambda \dot{\xi}_{t}} d t\right)\right] .
$$

On the other hand, by the uniform converge theorem for $\varphi$, see Theorem 1.5.2 of [5], we have $\varphi(x+y) / \varphi(x) \rightarrow e^{-\lambda y}$ as $x \rightarrow \infty$, uniformly for $y \in[0, \infty)$.

Applying (2.7) and the facts above to (4.23), we complete the proof.

For the proof of Theorem 3.6, we follow the same idea from [12] and [1].

Proof of Theorem 3.6. The theorem is proved by first claiming that under $\mathbb{Q}_{x}^{\uparrow}$,

$$
\frac{\bar{X}\left(T_{\infty}^{+}-t\right)}{\varphi^{-1}(t)} \longrightarrow 1 \text { as } t \rightarrow 0+,
$$

recalling that $\bar{X}(t):=\sup _{s \in[0, t]} X_{s}$ represents the running maximum process of $X$. The desired conclusion then follows from Lemma 2.1.

We first prove the statement (a). For any $h>1$, we take a constant $c_{h} \in(1$, $\left.\liminf _{y \rightarrow \infty} \frac{\varphi(y)}{\varphi(h y)}\right)$. By the result of the statement A of Theorem 3.3, for any $\varepsilon>0$ there is $k_{3}>0$ such that

$$
\mathbb{P}_{x}^{\uparrow}\left(\frac{J\left(\tau_{z}^{+}\right)}{\varphi(z)} \notin\left(c_{h}^{-1}, c_{h}\right)\right) \leq \varepsilon \quad \text { and } \quad 1<c_{h} \leq \frac{\varphi(z)}{\varphi(h z)} \quad \text { for } z>k_{3} .
$$


Set $t_{0}:=\varphi\left(h k_{3}\right)$. For $t<t_{0}$, define $\alpha(t):=h \cdot \varphi^{-1}(t)$ and $\beta(t):=\varphi^{-1}(t) / h$, then $\alpha(t)>\varphi^{-1}(t)>\beta(t)>k_{3}$. By the second inequality in (4.25),

$$
\frac{\varphi\left(\varphi^{-1}(t)\right)}{\varphi(\beta(t))} \leq c_{h}^{-1}<1<c_{h} \leq \frac{\varphi\left(\varphi^{-1}(t)\right)}{\varphi(\alpha(t))} .
$$

Then we further have from $\beta(t)>k_{3}$ that the following inequalities hold,

$$
\begin{aligned}
& \mathbb{P}_{x}^{\uparrow}\left(J\left(\tau_{\alpha(t)}^{+}\right) \geq t\right)=\mathbb{P}_{x}^{\uparrow}\left(\frac{J\left(\tau_{\alpha(t)}^{+}\right)}{\varphi(\alpha(t))} \geq \frac{\varphi\left(\varphi^{-1}(t)\right)}{\varphi(\alpha(t))}\right) \leq \mathbb{P}_{x}^{\uparrow}\left(\frac{J\left(\tau_{\alpha(t)}^{+}\right)}{\varphi(\alpha(t))} \geq c_{h}\right) \leq \varepsilon, \\
& \mathbb{P}_{x}^{\uparrow}\left(J\left(\tau_{\beta(t)}^{+}\right) \leq t\right)=\mathbb{P}_{x}^{\uparrow}\left(\frac{J\left(\tau_{\beta(t)}^{+}\right)}{\varphi(\beta(t))} \leq \frac{\varphi\left(\varphi^{-1}(t)\right)}{\varphi(\beta(t))}\right) \leq \mathbb{P}_{x}^{\uparrow}\left(\frac{J\left(\tau_{\beta(t)}^{+}\right)}{\varphi(\beta(t))} \leq c_{h}^{-1}\right) \leq \varepsilon
\end{aligned}
$$

which, since $\varphi\left(\varphi^{-1}(t)\right)=t$, gives for $t<t_{0}$

$$
\mathbb{Q}_{x}^{\uparrow}\left(h^{-1} \leq \frac{\bar{X}\left(T_{\infty}^{+}-t\right)}{\varphi^{-1}(t)} \leq h\right)=\mathbb{P}_{x}^{\uparrow}\left(J\left(\tau_{\alpha(t)}^{+}\right) \leq t \leq J\left(\tau_{\beta(t)}^{+}\right)\right) \geq 1-2 \varepsilon .
$$

We can prove the weak limit (4.24) by first letting $\varepsilon \rightarrow 0+$ and then letting $h \rightarrow 1+$.

For the statement (b), notice that for every $k_{4}>0$

$$
\frac{\varphi(z)}{\varphi(h z)} \geq \frac{\varphi(z)}{\varphi\left(z+k_{4}\right)} \rightarrow e^{\lambda k_{4}} \quad \text { as } z \rightarrow \infty .
$$

Thus, we always have $\liminf _{z \rightarrow \infty} \frac{\varphi(z)}{\varphi(h z)}=\infty$ for all $h>1$ in this case. Since $J\left(\tau_{z}^{+}\right) / \varphi(z)$ converges in law to a random variable on $(0, \infty)$, there exist $M>1$ and $k_{5}>0$ such that

$$
\mathbb{P}_{x}^{\uparrow}\left(\frac{J\left(\tau_{z}^{+}\right)}{\varphi(z)} \notin\left(\frac{1}{M}, M\right)\right) \leq \varepsilon \quad \text { and } \quad 1<M \leq \frac{\varphi(z)}{\varphi(h z)} \quad \text { for all } z>k_{5},
$$

which can be compared with (4.25). The same argument as in the previous case can be applied to prove (4.24). Applying the result of (C) in Proposition 4.10, we finish the proof.

\section{References}

[1] Bansaye, V., Méléard, S. and Richard, M.(2016). Speed of coming down from infinity for birth-and-death processes. Advances in Applied Probability, 48(4):1183-1210. MR3595771

[2] Bertoin, J. (1996). Lévy Processes. Cambridge Tracts in Mathematics. MR1406564

[3] Bertoin, J., van Harn, K. and Steutel, F. W. (1999). Renewal theory and level passage by subordinators. Statistics and Probability Letters, 45(1):65-69. MR1718352

[4] Bertoin, J. and Savov, M. (2011). Some applications of duality for Lévy processes in a half-line. Bulletin of the London Mathematical Society, 43(1):97-110. MR2765554

[5] Bingham, N. H., Goldie, C. M. and Teugels, J. L. (1987). Regular Variation. Cambridge: Cambridge University Press. MR1015093

[6] Bogachev, V. (2007). Measure Theory. Springer-Verlag Berlin Heidelberg. MR2267655

[7] Chen, A. (2002). Uniqueness and extinction properties of generalised Markov branching processes. Journal of Mathematical Analysis and Applications, 274: 482-494. MR1936711

[8] Chen, A.Y., Li, J.P. and Ramesh, N.I. (2008). Probabilistic approach in weighted Markov branching processes. Statistics and Probability Letters, 78: 771-779. MR2409542

[9] Dawson, D.A. and Li, Z. (2006). Skew convolution semigroups and affine Markov processes. Annals of Probability, 34: 1103-1142. MR2243880

[10] Döring, L. and Kyprianou, A. E. (2016). Perpetual Integrals for Lévy Processes. Journal of Theoretical Probability, 29: 1192-1198. MR3540494 
[11] Duhalde, X., Foucart, C. and Ma, C. (2014). On the hitting times of continuous-state branching processes with immigration. Stochastic Processes and their Applications, 124(12):4182-4201. MR3264444

[12] Foucart, C., Li, P.-S. and Zhou, X. (2021). Time-changed spectrally negative Lévy processes starting from infinity, Bernoulli, 27(2): 1291-1318. MR4255235

[13] Hubalek, F. and Kyprianou, E. (2011). Old and New Examples of Scale Functions for Spectrally Negative Lévy Processes. In Dalang, R., Dozzi, M., and Russo, F., editors, Seminar on Stochastic Analysis, Random Fields and Applications VI, volume 63 of Progress in Probability, pages 119-145. Springer Basel. MR2857022

[14] Jacobsen, M. and Jensen, A. T. (2007). Exit times for a class of piecewise exponential Markov processes with two-sided jumps. Stochastic Processes and their Applications, 117(9):13301356. MR2343943

[15] Klebaner, F. C. (1984). Geometric rate of growth in population-size-dependent branching processes. Journal of Applied Probability, 21: 40-49. MR0732669

[16] Kolb, M. and Savov, M. (2020). A characterization of the finiteness of perpetual integrals of Lévy processes. Bernoulli, 26(2):1453-1472. MR4058374

[17] Kuznetsov, A., Kyprianou, A. E. and Rivero, V. (2012). The Theory of Scale Functions for Spectrally Negative Lévy Processes. In Lévy Matters II, Lecture Notes in Mathematics, pages 97-186. Springer Berlin Heidelberg. MR3014147

[18] Kyprianou, A. E. (2014). Fluctuations of Lévy Processes with Applications. Springer Berlin Heidelberg. MR3155252

[19] Li, B. and Palmowski, Z. (2018). Fluctuations of Omega-killed spectrally negative Lévy processes. Stochastic Processes and their Applications, 128(10):3273-3299. MR3849809

[20] Li, B. and Zhou, X. (2019). Local Times for Spectrally Negative Lévy Processes. Potential Analysis, 52(4): 689-711. MR4091599

[21] Li, P.-S. (2019). A continuous-state polynomial branching process. Stochastic Processes and their Applications, 129 (8): 2941-2967. MR3980150

[22] Li, P.-S., Yang, X. and Zhou, X. (2019). A general continuous-state nonlinear branching process. Annals of Applied Probability, 29(4): 2523-2555. MR3983343

[23] Li, P.-S. and Zhou, X. (2018). Integral functionals for spectrally positive Lévy processes. arXiv:1809.05759

[24] Li, Z. (2012). Continuous-state branching processes. arXiv:1202.3223.

[25] Sevast'janov, B. A. and Zubkov, A.M. (1974). Controlled branching processes. Theory of Probability \& Its Applications, 19: 15-25. MR0339350

Acknowledgments. The authors thank an anonymous referee for detailed comments and helpful suggestions. 


\section{Electronic Journal of Probability Electronic Communications in Probability}

\section{Advantages of publishing in EJP-ECP}

- Very high standards

- Free for authors, free for readers

- Quick publication (no backlog)

- Secure publication $\left(\mathrm{LOCKSS}^{1}\right)$

- Easy interface (EJMS²)

\section{Economical model of EJP-ECP}

- Non profit, sponsored by $\mathrm{IMS}^{3}, \mathrm{BS}^{4}$, ProjectEuclid ${ }^{5}$

- Purely electronic

\section{Help keep the journal free and vigorous}

- Donate to the IMS open access fund ${ }^{6}$ (click here to donate!)

- Submit your best articles to EJP-ECP

- Choose EJP-ECP over for-profit journals

\footnotetext{
${ }^{1}$ LOCKSS: Lots of Copies Keep Stuff Safe http://www. lockss.org/

${ }^{2}$ EJMS: Electronic Journal Management System http://www.vtex.lt/en/ejms.html

${ }^{3}$ IMS: Institute of Mathematical Statistics http://www.imstat.org/

${ }^{4}$ BS: Bernoulli Society http://www. bernoulli-society.org/

${ }^{5}$ Project Euclid: https://projecteuclid.org/

${ }^{6}$ IMS Open Access Fund: http://www.imstat.org/publications/open.htm
} 